\title{
Dopamine Transporter Is a Master Regulator of Dopaminergic Neural Network Connectivity
}

\author{
Douglas R. Miller, ${ }^{1}$ Dylan T. Guenther, ${ }^{1}{ }^{\mathbb{D}}$ Andrew P. Maurer, ${ }^{1}$ Carissa A. Hansen, ${ }^{1}$ Andrew Zalesky, ${ }^{2,3}$ and \\ ${ }^{-}$Habibeh Khoshbouei ${ }^{1}$ \\ ${ }^{1}$ Department of Neuroscience, University of Florida, Gainesville, Florida, ${ }^{2}$ Melbourne Neuropsychiatry Centre, The University of Melbourne and \\ Melbourne Health, Melbourne, Victoria 3010, Australia, and ${ }^{3}$ Department of Biomedical Engineering, Melbourne School of Engineering, The \\ University of Melbourne, Melbourne, Victoria 3010, Australia
}

\begin{abstract}
Dopaminergic neurons of the substantia nigra pars compacta (SNC) and ventral tegmental area (VTA) exhibit spontaneous firing activity. The dopaminergic neurons in these regions have been shown to exhibit differential sensitivity to neuronal loss and psychostimulants targeting dopamine transporter. However, it remains unclear whether these regional differences scale beyond individual neuronal activity to regional neuronal networks. Here, we used live-cell calcium imaging to show that network connectivity greatly differs between SNC and VTA regions with higher incidence of hub-like neurons in the VTA. Specifically, the frequency of hub-like neurons was significantly lower in SNC than in the adjacent VTA, consistent with the interpretation of a lower network resilience to SNC neuronal loss. We tested this hypothesis, in DAT-cre/loxP-GCaMP6f mice of either sex, when activity of an individual dopaminergic neuron is suppressed, through whole-cell patch clamp electrophysiology, in either SNC or VTA networks. Neuronal loss in the SNC increased network clustering, whereas the larger number of hub-neurons in the VTA overcompensated by decreasing network clustering in the VTA. We further show that network properties are regulatable via a dopamine transporter but not a $\mathrm{D} 2$ receptor dependent mechanism. Our results demonstrate novel regulatory mechanisms of functional network topology in dopaminergic brain regions.
\end{abstract}

Key words: complex network analysis; dopamine; dopamine neurons; dopamine transporter; substantia nigra; ventral tegmental area

Significance Statement

In this work, we begin to untangle the differences in complex network properties between the substantia nigra pars compacta (SNC) and VTA, that may underlie differential sensitivity between regions. The methods and analysis employed provide a springboard for investigations of network topology in multiple deep brain structures and disorders.

\section{Introduction}

Dopaminergic neurons are biochemically (Poulin et al., 2014; Tiklová et al., 2019), structurally (Margolis et al., 2006; Fu et al., 2012), and functionally heterogeneous (Brown et al., 2009; Morales and Margolis, 2017; Saunders et al., 2018) and involved in a constellation of behaviors such as movement and valuebased choice (Schultz, 1986; Lerner et al., 2015; Beeler and

Received Jan. 29, 2021; revised Apr. 19, 2021; accepted May 1, 2021.

Author contributions: D.R.M. and H.K. designed research; D.R.M., D.T.G., and C.A.H. performed research; D. R.M., D.T.G., A.P.M., and A.Z. analyzed data; D.R.M. and H.K. wrote the paper.

D.R.M. was supported by the National Institute of Neurological Disorders and Stroke Grant T32-NS082128. H.K. was supported by National Institute on Drug Abuse and National Institute of Neurological Disorders and Stroke Grants R01-DA026947 and R01-NS071122. We thank Dr. Jeff Beeler for his insightful comments that improved the quality of the manuscript.

The authors declare no competing financial interests.

Correspondence should be addressed to Habibeh Khoshbouei at habibeh@ufl.edu.

https://doi.org/10.1523/JNEUROSCI.0223-21.2021

Copyright $\odot 2021$ the authors
Mourra, 2018; Beeler and Dreyer, 2019). Transcriptomic and molecular studies have identified multiple distinct classes of dopaminergic neurons that exist within the same anatomic region (Seroogy et al., 1988; Jayaraman et al., 1990; Rogers, 1992; Yamada et al., 1995; Liang et al., 1996; Numan and Seroogy, 1999; Neuhoff et al., 2002; Figlewicz et al., 2003; Korotkova et al., 2003; Cruz et al., 2004; Wang and Morales, 2008; Grieder et al., 2014; Poulin et al., 2014, 2018; Cork et al., 2015; La Manno et al., 2016; Pandit et al., 2016; Saunders et al., 2018; Tiklová et al., 2019). Morphologic investigations revealed a diverse and complex variation in the structural compartmentalization of dopamine neurons within each anatomic cluster.

The substantia nigra (SN; A9) and adjacent ventral tegmental area (VTA; A10) harbor two major dopaminergic clusters (Margolis et al., 2006; Fu et al., 2012). Despite their proximity, these two ventral midbrain regions contain strikingly different biological attributes, dopaminergic morphologies and protein expression patterns (Margolis et al., 2006; Fu et al., 2012; Poulin 
et al., 2014; Tiklová et al., 2019). While these differences have been hypothesized to underlie differential sensitivity to neurotoxins (Mattammal et al., 1995; Mosharov et al., 2009; Martin et al., 2016; Knowlton et al., 2018), drugs of abuse (Margolis et al., 2006; Hnasko et al., 2010; Lüscher, 2016; Morales and Margolis, 2017), and neurodegeneration (Braak and Braak, 2000; St Martin et al., 2007; Branch et al., 2014; Howell et al., 2020; Lin et al., 2020), it remains unclear how these differences manifest within the VTA and SN regional networks.

Furthermore, dopaminergic neurons vary in their intrinsic electrophysiological properties (Grace and Bunney, 1983; Rayport et al., 1992; Morales and Margolis, 2017), such as spontaneous firing rate, excitability, and spike duration, suggesting that individual neurons may differentially contribute to the synchronization of activity in dopaminergic networks. However, it remains unknown how networks arise from local interactions of dopaminergic neurons, what properties these regional neuronal networks exhibit, whether they differ by specific midbrain regional [VTA vs SN pars compacta (SNC)] identity, how they are regulated or fluctuate in time.

Recently, network neuroscience has emerged as an important tool to answer integral questions about brain network organization (Bassett and Sporns, 2017). Previous work using network theory found that densely connected neurons (hubs) not only exist but orchestrate synchrony (Sporns et al., 2007; Yu et al., 2008; Bonifazi et al., 2009; Sporns, 2013). Recent works have suggested that dopamine network synchrony drives interregional connectivity and whole brain synchronization (Beeler and Dreyer, 2019; Palacios et al., 2019). However, dopaminergic networks have yet to be investigated for the relative impact of the activity of individual neurons to network topology. Hubs, or highly connected neurons, are a common feature of complex networks (Sporns et al., 2007; Sporns, 2013; van den Heuvel and Sporns, 2013; Gutierrez and Marder, 2014; Schroeter et al., 2015), but dopaminergic networks have yet to be tested empirically for the existence of hub neurons. Consistently, many investigations describe the SNC dopaminergic network as more vulnerable to neuronal loss, as often observed in Parkinson's disease, than the adjacent VTA, albeit with a less understood mechanism (Sulzer, 2007; Boger et al., 2008; Mosharov et al., 2009; Vaillancourt et al., 2009; Evans et al., 2017; Lieberman et al., 2017; Tarfa et al., 2017; Fernandes et al., 2020b; Giguère et al., 2019). While the origin of these regional differences has remained nebulous, network analysis can provide clues to how functional network organization of these regions may contribute to resilience or failure within the network that ultimately lead to neurocognitive and behavioral dysfunction.

The dopamine transporter, the spatial and temporal regulator of dopamine signaling (Prasad and Amara, 2001; Ingram et al., 2002; Beckstead et al., 2004; Kahlig et al., 2005; Fog et al., 2006; Schmitt and Reith, 2010; Lin et al., 2016), which is expressed in nearly every dopamine neuron in both VTA and SN, providing a unique modicum of communication that is specific to monoamine systems. It has been shown previously that stimulation of dopamine transporter induces alteration in local network properties in reduced systems (Miller et al., 2019), and repeated exposure to modifiers of dopaminergic signaling, such as psychostimulants, regulates dopaminergic signaling machinery and brain activity (Sandoval et al., 2000; Volkow et al., 2001a,b, 2002; Wang et al., 2004; Kahlig et al., 2006; Chang et al., 2008; Krasnova et al., 2011; Volkow and Morales, 2015; Miller et al., 2019). Dopaminergic signaling relies heavily on volume transmission (Rice, 2000; Cragg et al., 2001; Beckstead et al., 2004; Cragg and Rice, 2004; O’Neill et al., 2017), enabling communication between both adjacent and distant neurons (Pucak and Grace, 1994; Cragg et al., 2001; Gentet and Williams, 2007; Gantz et al., 2013; Rice and Patel, 2015). Termination of the dopaminergic signal is thereby achieved by reuptake through dopamine transporter. However, it remains unexplored how dopamine transporter activity regulates interactions between dopaminergic neurons, leading to overall regulation of functional connectivity and ultimately dopaminergic network properties within the SNC and VTA. Previous models of dopaminergic networks suggest that information is encoded through either firing rate or synchronized activity (Beeler and Dreyer, 2019; Kremer et al., 2020; Starkweather and Uchida, 2020). However, these models do not explain whether dopamine transporter function is critical to the functional structure of dopaminergic networks, or whether perturbations of dopamine transporter activity result in restructuring of the network.

In this work, we used high speed live-cell calcium imaging to investigate dynamic functional connectivity and network properties of the SNC and VTA. Moreover, we determined regional-specific contributions of individual neurons to dopaminergic networks through electrophysiological manipulations and identified the role of dopamine transporter to the regulation of dopaminergic networks. We found that dopaminergic neurons in both VTA and SNC exhibit dynamic activity with similar magnitudes and variance of functional connectivity, but the incidence of hub-like neurons was significantly lower in SN dopamine neurons than in the adjacent VTA. Consequently, suppression of activity of an individual dopaminergic neuron within the SNC network increased network clustering, which measures triplet interactions important for functional integration, whereas it decreased network clustering in the VTA. Furthermore, we found that methamphetamine stimulation of dopamine transporter alters network assortativity and small-world properties, which can contribute to network vulnerability to insults like aberrant protein aggregation or neurotoxins. These results suggest that the regional heterogeneities of dopaminergic networks influence functional network topology. Furthermore, differences in functional network topology contribute or ameliorate network dysfunction in the presence of neuronal loss, and whether networks can be controlled through dopamine transporter regulation. This work begins to untangle the differences in complex network properties between the SNC and VTA, that may underlie differential sensitivity between regions and provides a springboard to investigations of multiple deep brain structures and disorders.

\section{Materials and Methods}

Animals

DAT $^{\text {IREScre }}$ and Ai95(RCL-GCaMP6f)-D knock-in mice were obtained from The Jackson Laboratory [stock numbers: 006660 (DAT ${ }^{\text {IREScre }}$ ), 024105 (Ai95D)]. Only animals exhibiting bright EGFP fluorescence were used for experiments. Approximately equal numbers of males and females were used for each experimental condition. All animals were maintained in the University of Florida animal facilities. All experiments were approved by the Institutional Animal Care and Use Committee at University of Florida. Animals were housed under standard conditions of $22-24^{\circ} \mathrm{C}, 50-60 \%$ relative humidity, and a $12 / 12 \mathrm{~h}$ light/dark cycle. 
Preparation of acute midbrain slices in imaging

Postnatal days 28-42 DAT-cre/loxP-GCaMP6f mice of either sex were used. Mice were decapitated and brain extracted immediately into icecold oxygenated artificial CSF (ACSF)s containing $126 \mathrm{~mm} \mathrm{NaCl}$, $2.5 \mathrm{~mm} \mathrm{KCl}, 2 \mathrm{~mm} \mathrm{CaCl}, 26 \mathrm{~mm} \mathrm{NaHCO}_{3}, 1.25 \mathrm{~mm} \mathrm{NaH}_{2} \mathrm{PO}_{4}, 2$ $\mathrm{mM} \mathrm{MgSO}_{4}$, and $10 \mathrm{~mm}$ dextrose, equilibrated with $95 \% \mathrm{O}_{2}-5 \%$ $\mathrm{CO}_{2}, 300-310 \mathrm{mOsm}$, and $\mathrm{pH}$ adjusted to 7.35-7.4. Brain was cut rostral to $\mathrm{PFC}$ in the coronal plane to create a flat surface to glue to the cutting stage. Brain was then submersed in fresh ice-cold ACSF as previously described; 200 - to $300-\mu \mathrm{m}$ sections containing either the SN or VTA were cut using a DSK MicroSlicer Zero $1 \mathrm{~N}$ and transferred to a recovery chamber at $32-34^{\circ} \mathrm{C}$ and allowed to recover for 45-60 min and maintained at room temperature (22$24^{\circ} \mathrm{C}$ ) until used.

\section{Electrophysiological recordings and analysis}

To facilitate electrophysiological recordings, the slicing preparation was modified with slicing performed in a sucrose-sodium-replacement solution containing $250 \mathrm{~mm}$ sucrose, $26 \mathrm{~mm}$ $\mathrm{NaHCO}_{3}, 2 \mathrm{~mm} \mathrm{KCl}, 1.2 \mathrm{~mm} \mathrm{NaH}_{2} \mathrm{PO}_{4}, 11 \mathrm{~mm}$ dextrose, $7 \mathrm{~mm}$ $\mathrm{MgCl}_{2}$, and $0.5 \mathrm{~mm} \mathrm{CaCl}$, and recovery (ACSF) was supplemented with $10 \mu \mathrm{M}$ MK-801 to reduce glutamate toxicity. Slices were transferred to a low-profile open diamond bath imaging chamber (RC-26GLP, Warner Instruments) under constant perfusion of ACSF (described previously) at $36-37^{\circ} \mathrm{C}$ at a rate of $2 \mathrm{ml} /$ min. Dopamine neuron cell bodies were identified first by GCaMP6f fluorescence through a $40 \times$ water immersion objective on an Eclipse FN1 (Nikon Instruments) upright microscope using a Spectra X light engine (Lumencor) equipped with a 470/24-nm solid-state illumination source and recorded using a 12-bit Zyla 4.2 sCMOS camera (Andor Technology, Ltd., Belfast, Northern Ireland). Neuronal morphology was then visualized using infrared differential interference contrast (IR-DIC). Borosilicate glass capillaries (1.5-mm O.D., Sutter Instrument Company) were pulled on a P-2000 laser puller (Sutter Instrument Company) to an open tip resistance of 4-10 $\mathrm{M} \Omega$ and filled with a potassium gluconate-based internal solution comprised $120 \mathrm{~mm} \mathrm{~K}$-gluconate, $20 \mathrm{~mm} \mathrm{KCl,} 2 \mathrm{~mm} \mathrm{MgCl}_{2}$, 10 mм HEPES, 0.1 mм EGTA, $2 \mathrm{~mm}$ $\mathrm{Na}_{2} \mathrm{ATP}, 0.25 \mathrm{~mm} \mathrm{NaGTP}$, and $5 \mathrm{~mm}$ biocytin, 290-295 mOsm, $\mathrm{pH}$ adjusted to 7.25-7.30. Recordings were made using an Axon Axopatch 200-B microelectrode amplifier and digitized with a Digidata 1440a through ClampEx 10.2 software (all Molecular Devices, LLC). Analysis of electrophysiological recordings was performed with custom code written for this project using Python 3.7 and the pyABF module (https://pypi.org/project/pyabf).

\section{Calcium imaging}

Acute coronal slices were illuminated as described previously (see above, Electrophysiological recordings and analysis). Images captured at 20-25 frames per second with no delay between frames. Videos were acquired either simultaneously or independent of electrophysiological recordings, depending on the experiment performed. Drug concentrations were selected after a careful review of the literature to model in vivo drug exposure in slice application (Ingram et al., 2002; Goodwin et al., 2009; Branch and Beckstead, 2012; Lin et al., 2016; Sambo et al., 2017; Miller et al., 2019). The literature suggests that $10 \mu \mathrm{M}$ methamphetamine evokes its maximum responses (Goodwin et al., 2009; Lin et al., 2016), which is blocked by $10 \mu \mathrm{M}$ nomifensine (Sambo et al., 2017).

Image correction, segmentation, and signal preprocessing

Nikon nd2 files were imported into ImageJ2 via the Bio-Formats importer. Motion correction was performed as necessary with the moco plugin (Dubbs et al., 2016). A rolling ball algorithm was applied to each image frame where a pixel value is determined by averaging a 50-pixel ball around the present pixel and then subtracted from the original image to reduce spatial variations. Cell bodies of spontaneously active dopamine neurons were manually segmented within clear borders region of interest (ROI), ensuring that ROIs did not overlap and exported to CSV. CSV files were imported into MATLAB and signals were smoothed using the loess method ( $\operatorname{span}=0.5 \%$ of data points). Signals were normalized using relative fluorescence by the following calculation:

$$
\Delta F / F_{0}=\frac{F_{t}-\tilde{F}_{t(1-25 s)}}{\tilde{F}_{t(1-25 s)}} .
$$

Where $F$ at time $t$ is normalized to the baseline measurement. The baseline is calculated as the median value of the first $25 \mathrm{~s}$ of the recording on a per neuron basis.

\section{Dynamic functional connectivity and network analysis}

Dynamic functional connectivity was computed using pairwise Spearman's correlation coefficients (MATLAB function: corr, type: Spearman) calculated over a sliding 100 frame window with a one frame step size between neurons. Since no threshold is privileged and arbitrary thresholds affect network measurements (Langer et al., 2013; Garrison et al., 2015; van den Heuvel et al., 2017), multiple proportional thresholds were applied to each correlation matrix from $5 \%$ to $100 \%$ at $1 \%$ intervals. Thresholds were excluded if all connections remaining were to one neuron or if less than four neurons remained in the network. For modularity and path length measurements, negative correlations were set to 0 to enable proper computation. All network analysis used the weighted function versions from the Brain Connectivity Toolbox (Rubinov and Sporns, 2010). Control weighted networks were created by randomizing the connection topology while maintaining the size, density, and degree distribution (Maslov and Sneppen, 2002) or latticized (Sporns and Kötter, 2004) where appropriate. Network metrics were calculated at each threshold and then averaged to give a timeresolved network metric that is balanced for specificity and sensitivity before moving to the next matrix.

For all networks, usage of node refers to a neuron. Normalized network strength was defined as the sum of all weights per neuron divided by the number of neurons in the observed network (Rubinov and Sporns, 2010) as defined by:

$$
S_{i}=\frac{\sum_{j} w_{\mathrm{ij}}}{N-1} .
$$

Importantly, normalized network strength was calculated using the full correlation matrix without thresholding.

Network assortativity, an evaluative measurement of preferential connectivity to neurons with similar or dissimilar connection strength (Newman, 2002; Foster et al., 2010; Teller et al., 2014; Miller et al., 2019). Assortativity $(r)$ was calculated as defined by:

$$
r(\alpha, \beta)=\frac{E^{-1} \sum_{i}\left[\left(j_{i}^{\alpha}-\overline{j^{\alpha}}\right)\left(k_{i}^{\beta}-\overline{k^{\beta}}\right)\right]}{\sigma^{\alpha} \sigma^{\beta}} .
$$

Where the nodal strength of neuron ja (source) and nodal strength of neuron $\mathrm{j} \beta$ (target) at connection $\mathrm{i}$ of all connections $(E)$. With $\sigma$ defined similarly for $\alpha$ and $\beta$ be:

$$
\sigma^{\alpha}=\sqrt{E^{-1} \sum_{i}\left(j_{i}^{\alpha}-\overline{j^{\alpha}}\right)^{2}}
$$

Network clustering, an extension on pairwise-correlation to triplet correlations (Chambers and MacLean, 2016; Miller et al., 2019), was determined by calculating clustering coefficients (Onnela et al., 2005). Clustering coefficients were calculated as defined by the following:

$$
\tilde{C}_{i}=\frac{2}{k_{i}\left(k_{i}-1\right)} \sum_{j, k}\left(\tilde{w}_{\mathrm{ij}} \tilde{w}_{\mathrm{jk}} \tilde{w}_{\mathrm{ki}}\right)^{\frac{1}{3}}
$$

Where $k_{i}$ is the degree of node $i$ and the weight $w$ between nodes $i$ and $\mathrm{j}$, computed across the triangle inclusion of nodes $\mathrm{j}, \mathrm{k}$. 
A

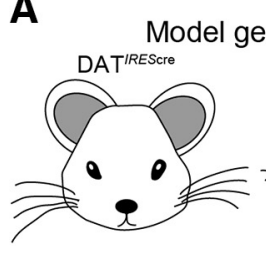

eneration

Ai95(RCL-GCaMP6f)
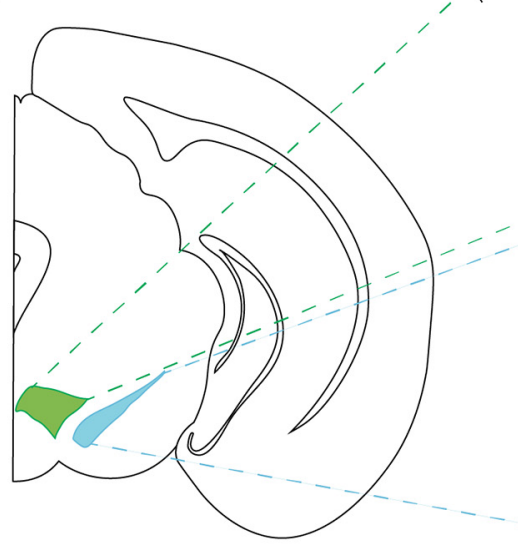

D

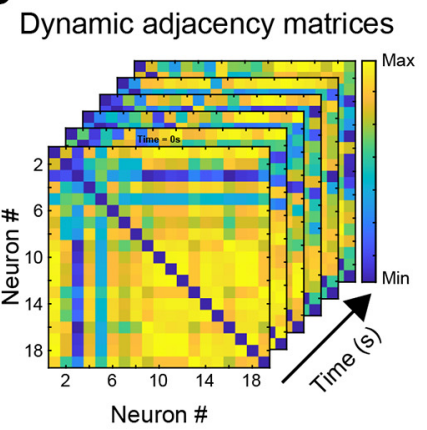

B

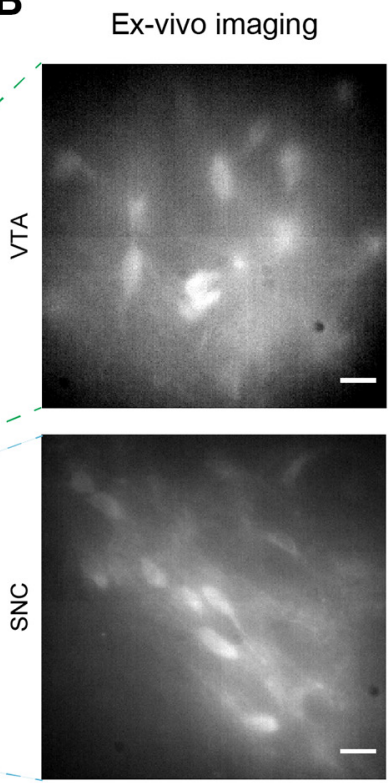

C
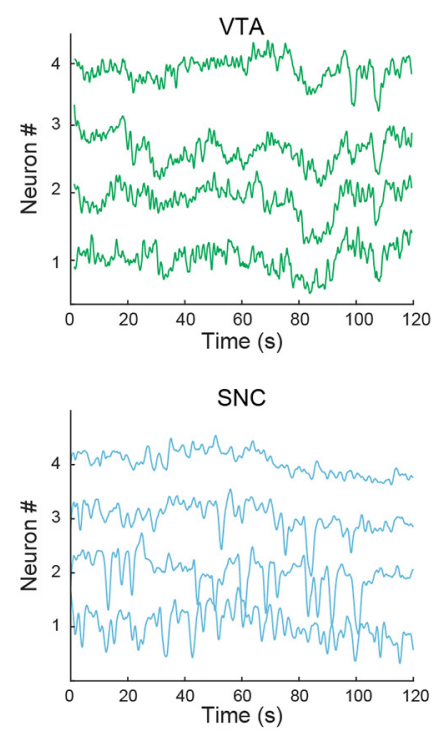

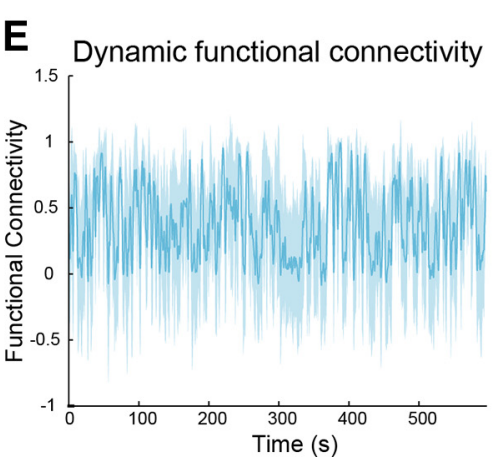

$\mathbf{F}$

Dynamic network properties

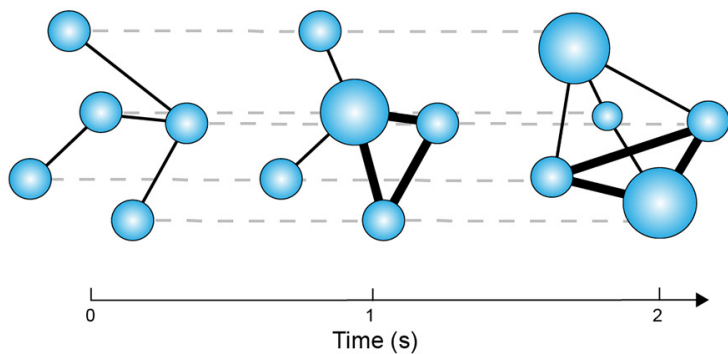

Figure 1. Schematic of experimental design and analytical pipeline to assay dynamic network properties in the VTA and SNC. Overview of experimental design and analytical processing. $A$, DAT ${ }^{\text {IREScre }}$ mice were crossed with Ai95(RCL-GCaMP6f) mice to yield mice expressing GCaMP6f in dopaminergic neurons of the ventral midbrain. $\boldsymbol{B}$, Imaging of spontaneous dopaminergic activity is performed in the SNC and VTA. Scale bar: $50 \mu \mathrm{m}$. C, Visibly active neurons were manually segmented as ROls and spontaneous neuronal calcium signal activity was extracted. $\boldsymbol{D}$, Pairwise correlation coefficients (Spearman's $\rho$ ) were calculated between each neuron of a sliding temporal window (100 frames, one frame step size) to generate dynamic adjacency matrix stack. $\boldsymbol{E}$, Permatrix averages were used to determine average functional connectivity over time. $\boldsymbol{F}$, Weighted networks, in which correlations between neurons are factored into network measurements, are constructed from dynamic adjacency matrices (D). To balance specificity (correlations higher than a percentile) and sensitivity (inclusion of weak correlations), network measurements were calculated over multiple thresholds, whereby $5 \%$ of the strongest correlations are retained for measurement. Thresholding proceeds in $1 \%$ increments until all correlations are retained (100\% threshold). Final measurements are then averaged across all thresholds to produce the mean network topology per time point. Circles represent individual neurons and their size corresponds to node strength in the network topology (larger $=$ more contributing). Connection thickness indicates correlation magnitude between neurons.

Characteristic path lengths, the average minimum number of connections between any two neurons in the network that must be traversed to connect them (Rubinov and Sporns, 2010), infinite path lengths were omitted from the calculations. Following the original definition by of a small-world network (Watts and Strogatz, 1998; Telesford et al., 2011), the small-world parameter $(\omega)$ was calculated as defined by the following:

$$
\omega=\frac{L_{r}}{L}-\frac{C}{C_{l}} .
$$

Where $\mathrm{L}$ and $\mathrm{C}$ refer to path length and clustering coefficient and $\mathrm{L}_{\mathrm{r}}$ is the path length of a weighted random lattice and $C_{l}$ is clustering of a weighted random network.

Network modularity, the existence of communities around neurons is quantified by the Q value index (Newman, 2006; Reichardt and Bornholdt, 2006).

Statistical analysis and network graph construction

All statistical analysis was performed in MATLAB (version 2020a, MathWorks). Two-sample $t$ tests were used where appropriate. Effects were determined as significant at the $\alpha=0.05$ level. Undirected network connections were imported with corresponding XY positions from the image plane into Gephi v0.9.2 (Bastian et al., 2009).

Data and code availability

All raw ROI fluorescence data, and processed functional connectivity and network metrics are provided in MAT files (MATLAB). Custom MATLAB code is intended to run as compiled code on a high-performance computing cluster. MATLAB livescript versions of the code, with raw and processed data, are available on request.

\section{Results}

Establishing a temporally and spatially-resolved model system to investigate the functional connectivity and network properties of dopaminergic neurons in the VTA and SNC

To investigate the intrinsic modulation of dynamic functional connectivity and properties of dopaminergic networks of the VTA and SNC, we used highspeed calcium imaging of neural activity (Fig. 1). Dopaminergic neurons signal through their canonical neurotransmitter, dopamine, at multiple release sites, 
notably through somatodendritic release via a calcium-dependent manner (Beckstead et al., 2004; Borisovska et al., 2013). Furthermore, dopaminergic neurons signal through multiple timescales (Beeler and Mourra, 2018; Berke, 2018), via presynaptic (Pucak and Grace, 1994; Benoit-Marand et al., 2001; Lindgren et al., 2001; Ingram et al., 2002; Branch and Beckstead, 2012; Dreyer and Hounsgaard, 2013; Rice and Patel, 2015) and postsynaptic mechanisms (Ingram et al., 2002; Lin et al., 2016; Aversa et al., 2018). While dopaminergic neurons receive diverse inputs into their respective regions, little is known about the self-organized intrinsic dopaminergic networks of the VTA and SNC. For these reasons, we used live-cell calcium imaging in acute midbrain slices spanning the VTA and SNC of mice, which provides a readout of neural activity that is intrinsically related to dopamine neuronal activity (Engelhard et al., 2019). We achieved high-specificity expression of GCaMP6f in the dopamine neurons, within these regions, by crossing a GCaMP6f reporter line with DAT-cre. The recordings were performed at $20-25 \mathrm{~Hz}$ with an average of 13 individually resolvable neurons per experiment. Thereby, our model system provides a rigorous approach to resolve dopamine neuronal activity with both high spatial and temporal resolution afforded by GCaMP6f kinetics, as well as recording from both SNC and VTA within the same slice.

Calcium influx and GCaMP6f responses exhibit a nonlinear relationship (Chen et al., 2013). Additionally, dopaminergic signaling occurs at multiple timescales, and synchronized neuronal activity is pivotal in dopamine communication (Beeler and Mourra, 2018; Berke, 2018; Beeler and Dreyer, 2019; Palacios et al., 2019). For these reasons, we used Spearman's $\rho$ on the calcium signal between every neuron within the field of view over a 100 -frame sliding window to construct a stack of adjacency matrices. Each matrix of correlation coefficients is then averaged to give the instantaneous functional connectivity of the network.

The time resolved adjacency matrices were then used to construct dynamic networks. Network measurements have previously been shown to be particularly sensitive to threshold values (Garrison et al., 2015; Zalesky et al., 2016), e.g., correlations that are believed to be meaningful. Importantly, there is no consensus on a meaningful correlation (Zalesky et al., 2016; van den Heuvel et al., 2017), i.e., a correlation of 0.2 could be as important as a value of 0.9 ; therefore, we strategized with a sliding threshold function where relative thresholds were applied starting at a retainer of $5 \%$ of the strongest correlations, to a fully connected network with all correlations retained, at $1 \%$ intervals. Network metrics were calculated at each threshold and then averaged to give a time-resolved network metric that is balanced for specificity and sensitivity before moving to the next matrix. This procedure enabled detailed and comprehensive investigation of the dynamic properties of dopaminergic networks of the VTA and $\mathrm{SNC}$, and how they are mechanistically regulated.

\section{Benchmarking temporal and spatial isolation of activity in individual neurons}

To better understand the accuracy of the manual segmentation on the spatial correlations between neurons, we generated a representative dataset with a laser scanned confocal reference volume of the region imaged in the calcium imaging dataset. In the laser scanning confocal image (Fig. $2 A$, left), neurons can easily be matched to the segmentation image from the calcium imaging dataset (Fig. 2A, right), with ROI boundaries non-overlapping of proximal neurons. Yellow arrows show neurons 7, 8, 9 that were selected for their high degree of spatial proximity and thus testing the accuracy of the manual segmentation on the spatial correlations between neurons. We considered these three neurons, because neuron 9 is situated between neuron 7 and neuron 8. ROI centroid distances between neuron 9 and neuron 7 is 43 pixels and for neuron 9 and neuron 8 the distance is 54 pixels. One would predict, by spatial proximity for the correlation between neuron 7 and neuron 9 to be significantly higher than neuron 8 and neuron 9. Therefore, we looked at the raw signals found from each neuron (Fig. $2 B$ ). Contrary to our prediction, we find that the correlation between neurons 7 and 9 to be 0.3696 , while the correlation between neurons 8 and 9 is 0.6202 despite neuron 7's ROI being closer to neuron 9's than neuron 8's (Fig. 2C). Thus, the analyses and the conclusions made here are not significantly affected by spatial correlation overlap.

We also noticed that the correlation between neuron 7 and neuron 8 are lower than other correlations. For instance, these two neurons are farther apart from each other than either from neuron 9, suggesting distance and correlation of activity might be related. Therefore, we examined the relationship between distance and correlation by considering the average correlation between neuronal pairs as a function of distance. Investigating analyses of spatial correlation between these neurons, did not reveal a strong correlation between distance and activity correlation $(r=-0.18, p=0.06$; Fig. $2 D)$. Therefore, in acute slice preparation, distance does not dictate degree of correlation as a regionally extrinsic process.

\section{SNC and VTA exhibit similar patterns of dynamic functional connectivity}

Dynamic functional connectivity quantifies the coupling between the time-series activity of a pair of neurons within a network as a function of time. Traditionally, these are applied to time-locked behavior events (Lurie et al., 2020). However, dopaminergic neurons exhibit tonic spontaneous firing activity that perseveres when excitatory neurotransmission is blocked (Bean, 2007; Puopolo et al., 2007; Lin et al., 2016), suggesting a basal pattern of activity occurs in each region. Here, we use correlated calcium signals to determine dynamic functional connectivity of SNC and VTA dopaminergic networks. First, we assayed the average functional connectivity (average of correlations between all neuronal pairs within the observed network) over the entire duration of the experiment within each region followed by analysis of the variance of this measurement across time to determine whether SNC and VTA exhibit similar functional connectivity over time. Surprisingly, the SNC and VTA exhibited similar average functional connectivity (two-tailed unpaired $t$ test, $p=0.7139, n=5-8$ sections from 5 animals; Fig. $3 B$ ), suggesting that functional connectivity alone does not sufficiently provide an understanding of differential vulnerability between VTA and SNC dopaminergic neurons.

We next asked whether these two regions would exhibit similar patterns in functional connectivity over time. To do so, we measured the coefficient of variation in the functional connectivity of each region and found similar patterns of functional connectivity within the SNC and VTA dopaminergic networks (twotailed unpaired $t$ test, $p=0.6887$; Fig. $3 C$ ). Similar average and dynamic functional connectivity patterns in the VTA and SNC dopamine neurons suggest that each region has similar basal oscillatory processes, which have been theorized to underlie how dopaminergic networks encode information (Beeler and Dreyer, 2019). The limitation of average functional connectivity is that it does not provide information about the structure of the functional network, and specific properties these may confer. 
A

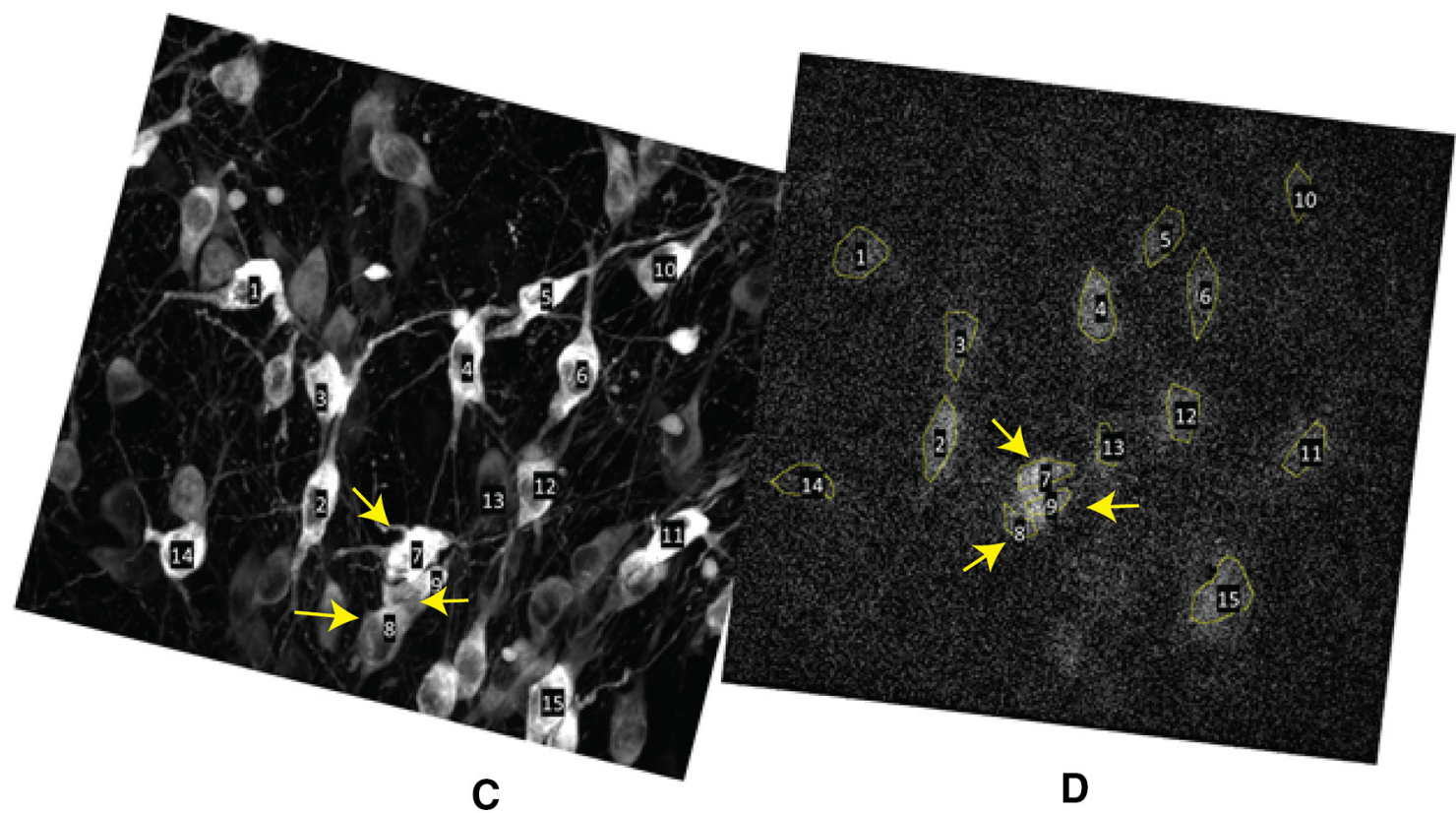

B

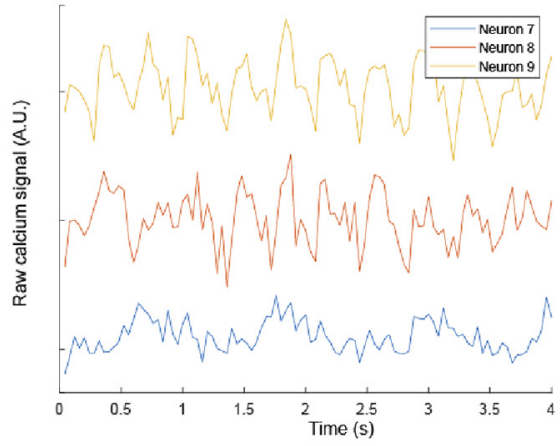

C

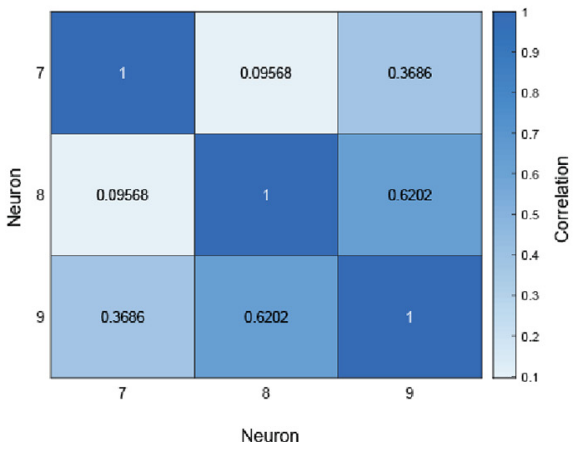

Relationship of correlation and distance

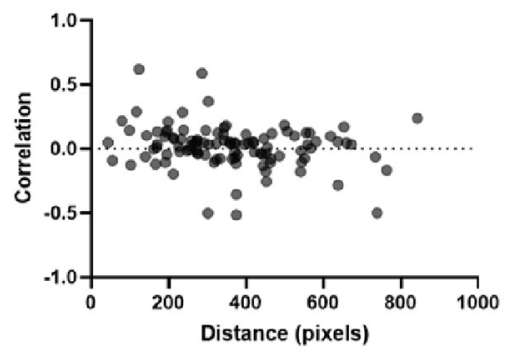

Figure 2. Representative benchmark of temporal and spatial isolation of calcium activity. A, 3D laser-scanning confocal image (left) of a VTA slice from a live-cell calcium imaging dataset (right). $\boldsymbol{B}$, Raw calcium activity from neurons 7,8 , and 9 (shown in $\boldsymbol{A}$ ) were selected for their high degree of spatial proximity. C, Correlation matrix of calcium activity between neurons 7,8 , and 9. $\boldsymbol{D}$, No significant relationship between neuronal soma distance and calcium activity is observed in the representative dataset.

In contrast to functional connectivity, complex network theory provides a well-accepted approach to investigate patterns of connectivity, which in turn facilitate or limit information transfer between dopamine neurons and across the network, that can reveal predisposition to failure, loss of information transfer within functional networks (Rubinov and Sporns, 2010; Bassett and Sporns, 2017). Therefore, we used the complex network analysis to examine the functional network properties of the SNC and VTA.

\section{Emergent variance of network hubs in the SNC and VTA}

To investigate patterns of functional network connectivity in each region, we examined the strength of connections (magnitude of the correlated activity between neurons) in the SNC and the VTA. Within the observable neurons of the field of view in each region, we found a wide distribution of connection strengths between dopamine neurons, where some neurons would exhibit low connectivity to each neuron within the network, while others would be strongly interconnected, suggesting that hub neurons may exist within the dopaminergic neuronal network in the VTA and SNC (Fig. 4A,B). Therefore, we calculated the nodal strength of each neuron by integrating the connection values to every other neuron per matrix and over time. Since more connections can arbitrarily inflate nodal strength values, temporal nodal strength means were normalized to the relative size of the network (Fig. $4 C$ ). Similar to the pattern of network connectivity of the network visualizations in Figure $4 A$, $B$, we found differential patterns of connectivity between dopaminergic neurons of the SNC and VTA. In the SNC, we found skewing of the connection strength distribution to lower strength values. Whereas, VTA neurons exhibited stronger connections across the network compared with the SNC [mean normalized node strength $(n)=3.97$ (89 neurons), SNC, 5.24 (70 neurons), VTA, two-tailed unpaired $t$ test, $\left.p=1.89 \times 10^{-9}\right]$. Because of the insurmountable technical restraints using brain slices, one of the limitations of our approach is that we have computed node strength in relatively small neuronal networks, in each brain structure, comprising 10-20 nodes. Nevertheless, even in this limited scale our analyses revealed the SNC to be less densely interconnected than the VTA, suggesting a potentially increased vulnerability of the region to insults.

The variances in nodal strength distributions between regions suggest that some neurons behave as network hubs, or neurons that significantly contribute more to the network than others. As nodal strength belongs to a group of network measurements known as nodal centrality, nodal strength provides a simplistic evaluation for hub-like properties of neurons within the region. We combined the weighted connection distributions (magnitude 

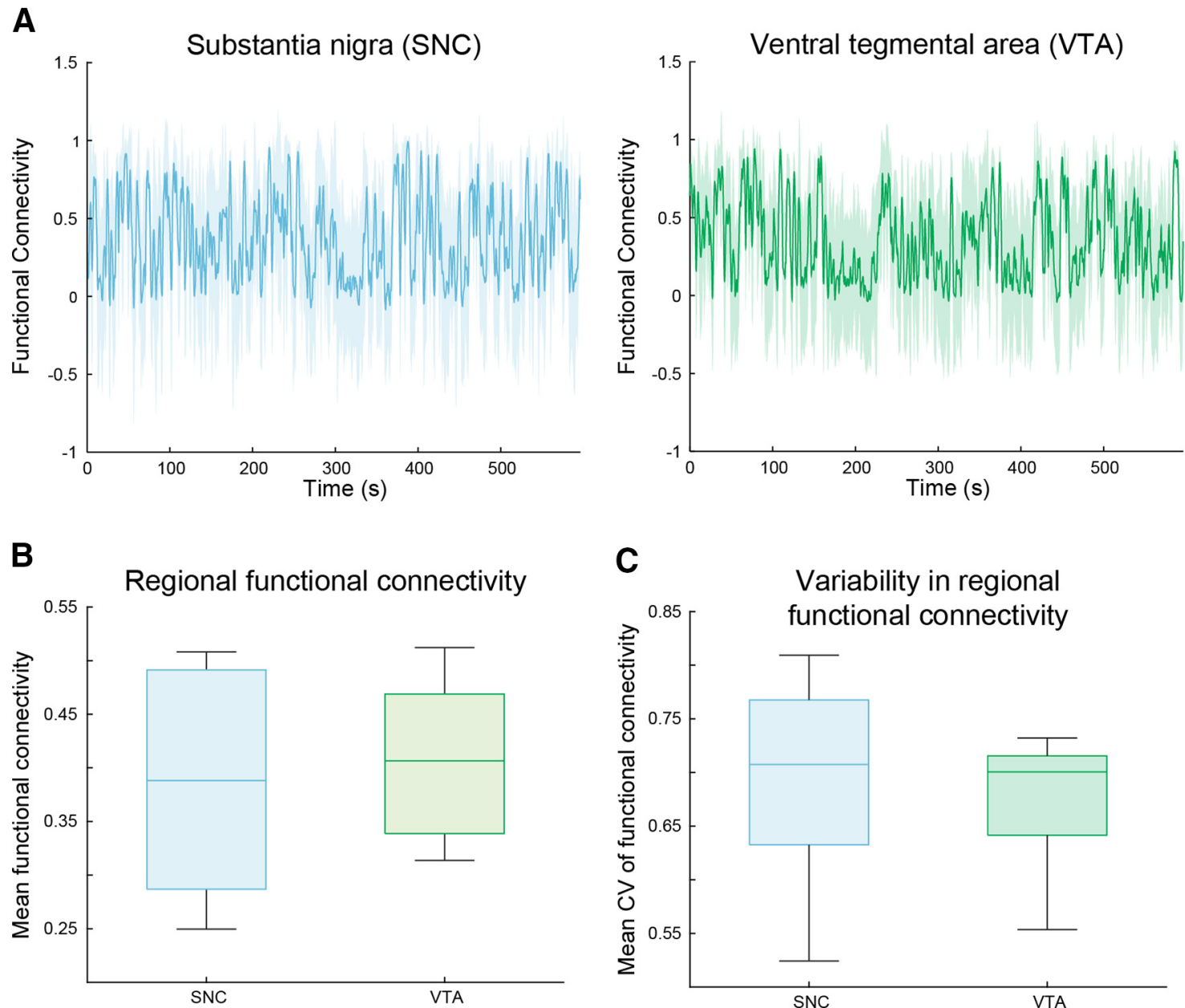

Figure 3. SNC and VTA exhibit similar patterns of dynamic functional connectivity. Intrinsic dynamic functional connectivity of dopamine neurons of the SN (SNC) and VTA exhibit similar patterns of functional connectivity. Functional connectivity is defined as the average pairwise Spearman's correlation across all neuronal pairs. $A$, Representative dynamic connectivity in the SN and VTA. $\boldsymbol{B}$. Mean functional connectivity is similar in the SN and VTA (mean FC $=0.386,0.407$ SNC, VTA, respectively, two-tailed unpaired $t$ test, $p=0.7193$ ). $\boldsymbol{C}$, Variability of functional connectivity (coefficient of variation, CV) is similar across the two regions (mean CV $=0.694,0.673$ SNC, VTA, respectively, two-tailed unpaired $t$ test, $p=0.6887$ ); $n=5-8$ sections from 5 animals.

of Spearman's $\rho$ between each neuron) of both regions to determine an average nodal strength value regardless of region, and calculated the relative percentages above and below the value for the SNC and VTA. Only a relatively small fraction of SNC neurons met this criterion $(32.58 \%)$ while the vast majority of neurons in the VTA $(78.57 \%)$ exceeded it (Fig. 4). These results suggest that loss of hub neurons in the SNC may have more drastic consequences to network properties and its potential lower resilience to failure than a similar loss in the VTA, potentially underlying the differential sensitivity ascribed to the two regions (Achard, 2006; Gao et al., 2016).

\section{Network properties of the SNC and VTA}

Similar to other brain networks (Sporns, 2011), it is likely that dopaminergic network connectivity is non-random in functional structure. However, functional network topology can emerge from randomly interacting neurons (Pernice et al., 2011; Orlandi et al., 2013). Therefore, we sought to determine first whether real network properties of the SNC and VTA are distinct from chance events. To determine whether the dynamic properties of each region deviate from a random network of equivalent size, each property was evaluated against a random weighted network (or random lattice, which is appropriate for small-world network measurements; Telesford et al.,
2011) of equivalent size and functional connectivity magnitude. Importantly, these random networks were generated through either a rewiring parameter performed for 1000 iterations randomly or with latticization (Maslov and Sneppen, 2002; Sporns and Kötter, 2004). Dopaminergic networks of the SNC and VTA were then assessed for network assortativity (preferential connectivity), modularity (delineation of clear non overlapping groups), path length (average network distance between nodes), and clustering coefficient (higher order interactions and functional segregation).

We first assayed network assortativity, which ascertains preferential attachment of a neuron to other neurons within the network. Specifically, if a neuron with many connections is primarily connected to other neurons with many connections (preferred connectivity to neurons with similar connectivity), that neuron will have a positive assortativity value, whereas if a neuron with many connections primarily connects to neurons with only one connection (preferred connectivity to neurons with dissimilar connectivity), that neuron will have a negativity assortativity value. Importantly, positively assortativity has previously been shown to provide robustness and resilience to networks (Teller et al., 2014; Lim et al., 2019), while negativity assortativity increases speed of information transfer (Gallos et al., 2008; Murakami et al., 2017). Therefore, examination of 
A

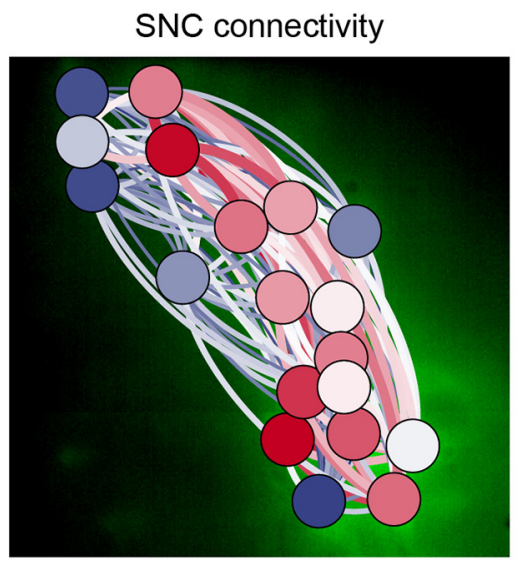

C

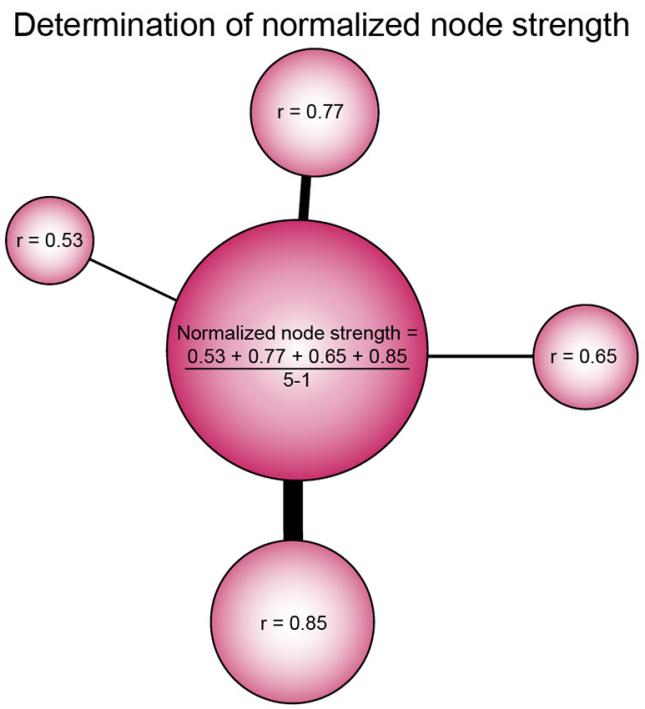

B

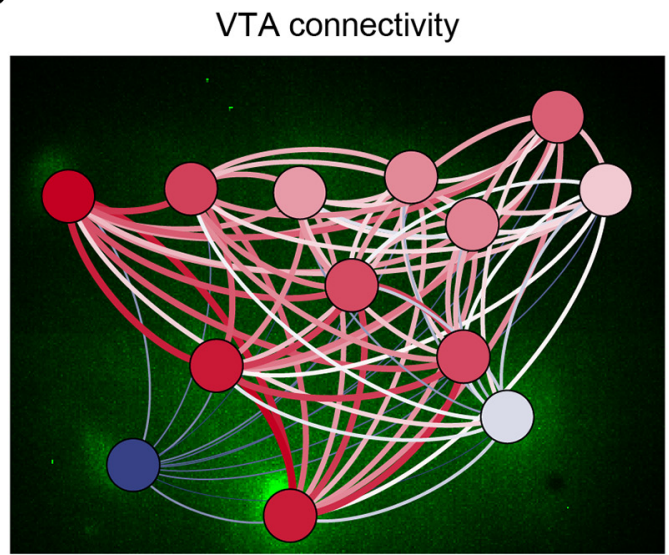

D

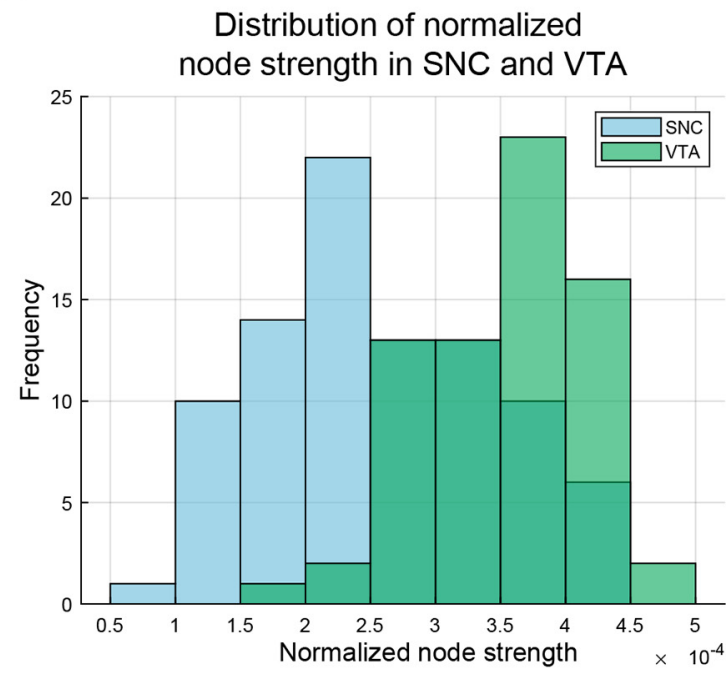

E

Relative percentage above average nodal strength

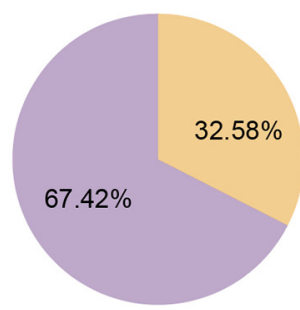

SNC

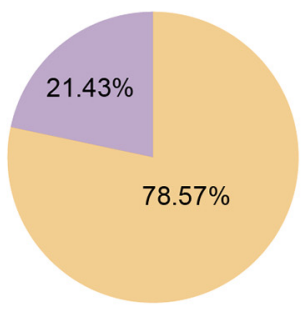

VTA
Percent above mean

Percent below mean

Figure 4. VTA dopaminergic networks are more strongly interconnected than the SNC. $\boldsymbol{A}, \boldsymbol{B}, \mathrm{XY}$-projection network graph of the functional connectivity matrices overlaid on SNC and VTA suggest differences in connection strength distributions. Connection strength, a neuron's relative contribution to the network, is displayed through coloration (blue $=$ minimum, white $=$ mean, red = high). Neurons are overlaid with circles representing their weighted degree (node strength, blue = minimum, white $=$ mean, red $=$ high). Scales reflect distribution within the investigated network. $\boldsymbol{C}$, Normalized node strength is determined through integrating the magnitude of the correlation values of each neuron to all other neurons within the network, and then normalized by dividing by $N-1$, where $N=$ total number of neurons within the network. $\boldsymbol{D}$, VTA neurons tend to exhibit larger normalized node strength values with a right skewed distribution while SNC exhibit a more Gaussian-like distribution centered on a lower value [mean normalized node strength $(n)=3.97$ ( 89 neurons), SNC, 5.24 (70 neurons), VTA, two-tailed unpaired $t$ test, $p=1.89 \times 10^{-9}$ ]. Node strengths are pooled for each region from five to eight slices from five animals. $\boldsymbol{E}$, Normalized node strength values were pooled for midbrain average node strength and then neurons were classified whether they exceeded or fell below this value. The VTA exhibits a significantly greater proportion of neurons exceeding this average midbrain node strength than SNC (78.57\%, VTA; 32.58\%, SNC; two-tailed unpaired $t$ test, $\left.p=3.80 \times 10^{-13}\right)$.

network assortativity provides critical information about resiliency and speed of information transfer in the VTA and SNC dopaminergic neurons/network. We found that the SNC and VTA dopaminergic networks both exhibit weakly positive assortativity (random networks generally exhibited negative assortativity), suggesting that neurons preferentially connect to neurons with similar connectivity properties that oscillate between positive and negative values over time (Fig. $5 A-C$ ).

We next quantified modularity of the network, or the degree to which neurons form specific groups that are more 
A

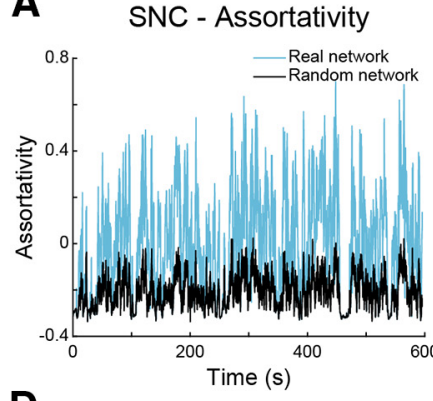

D

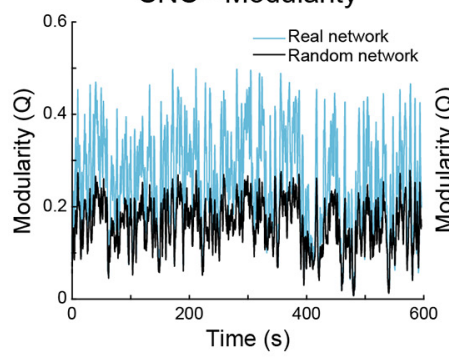

G

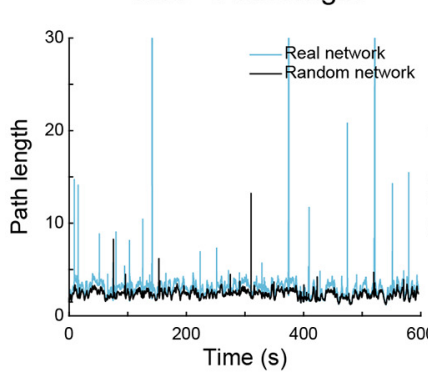

J SNC - Clustering coefficient

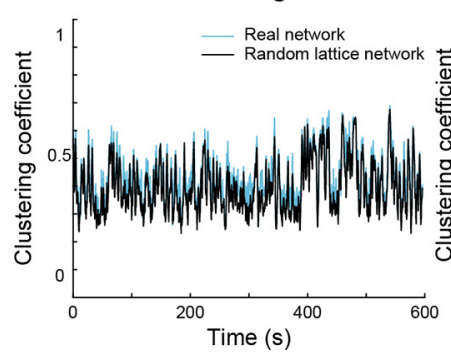

VTA - Assortativity

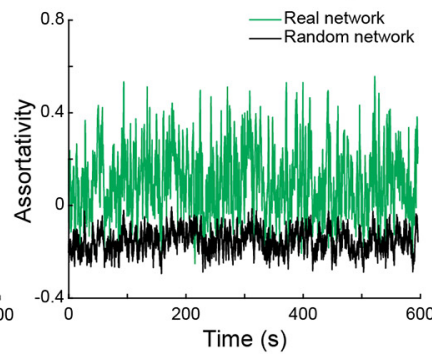

VTA - Modularity

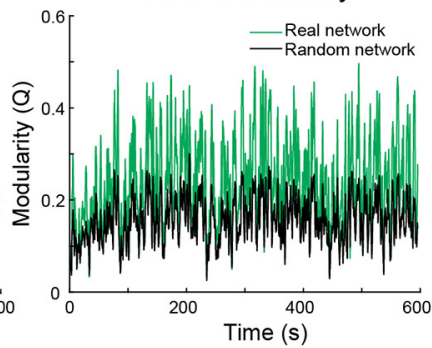

VTA - Path length

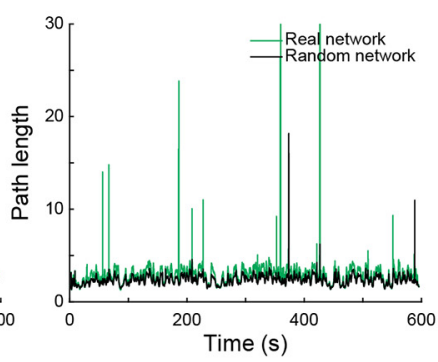

VTA - Clustering coefficient

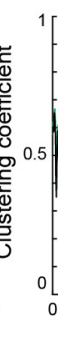

B

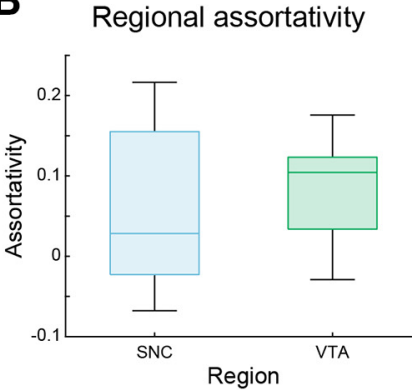

E

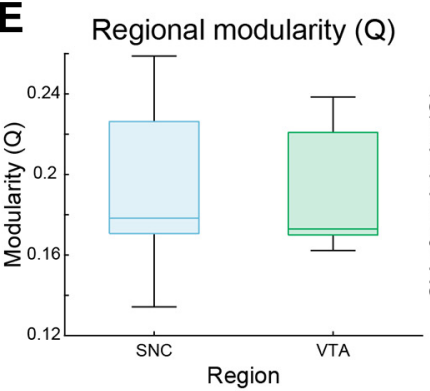

H
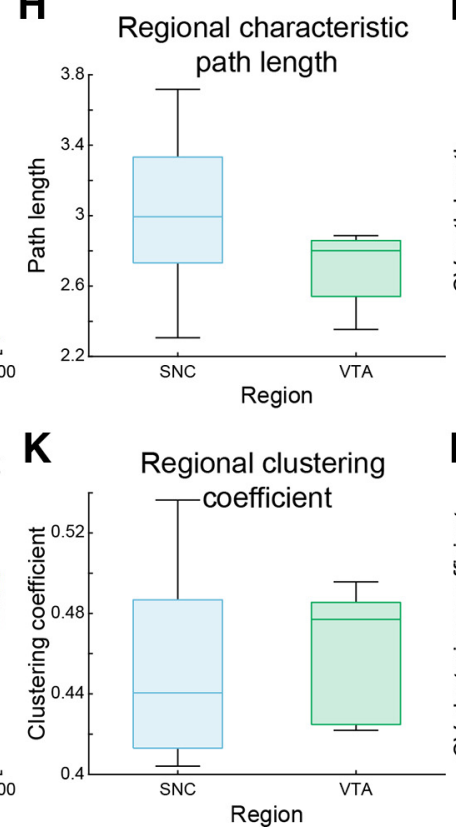

C Regional CV of assortativity

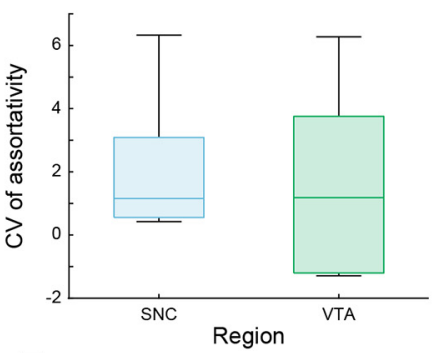

$F_{\text {Regional CV of modularity (Q) }}$

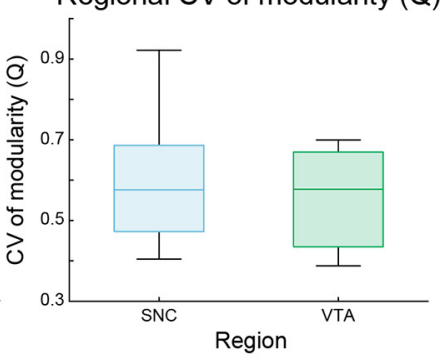

I

Regional CV characteristic

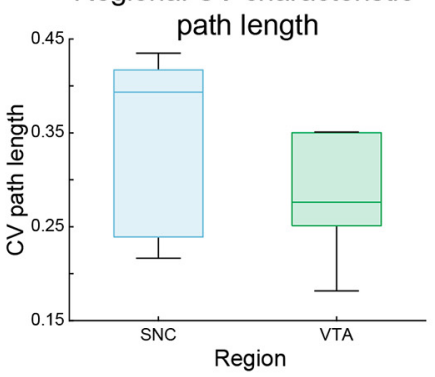

I Regional CV clustering

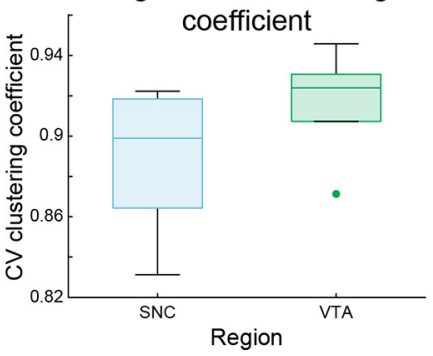

Figure 5. Complex network analysis reveals non-random ordered topology of SNC and VTA dopaminergic networks. We investigated regional dynamic network properties to determine whether they differ from those of a random network of equivalent size and connection distribution. Networks were assayed for $(A)$ assortativity, preferential connectivity of a neuron to other neurons with similar (positive) or dissimilar (negative) number of connections; $(\boldsymbol{D})$ modularity, the degree to which neurons form subspecialized groups; $(\boldsymbol{G})$ path length, the average number of connections separating any two neurons within the network; and $(\boldsymbol{l})$ clustering coefficient, a triplet interaction between three neurons. Across each measurement, SNC and VTA networks exhibited similar network assortativity $(\boldsymbol{B})$, modularity $(\boldsymbol{E})$, characteristic path length $(\boldsymbol{H})$, and clustering coefficient $(\boldsymbol{K})$ that were significant from their randomized counterparts and exhibited similar dynamics $(\boldsymbol{C}, \boldsymbol{F}, \boldsymbol{I}, \boldsymbol{L}) ; n=5-8$ sections.

interconnected with each other than neurons outside the group, ranging from 0 to 1 , where 0 represents no modular separation and 1 indicates a strong modular structure. Highly modular networks enable subspecialization of the neurons within a network (Sporns and Betzel, 2016; Paldino et al., 2017). Similarly, both regions exhibited equivalent non-random consistent modular functional structures (Fig. $5 D-F$ ), suggesting that modular segregation, and thereby subspecialization of neuronal groups, occurs consistently in the SNC and VTA. To investigate the relationship of robustness and efficiency of information transfer, we evaluated the network beyond pairwise comparisons to triplets, determined by clustering coefficients, which assays the connectivity between three neurons simultaneously (MacLean et al., 2005; Chambers and MacLean, 2016; Miller et al., 2019). High clustering coefficients are associated with network robustness (Bullmore and Sporns, 2009). We additionally quantified global efficiency using path length, where a low path length suggests that information can quickly move between clusters facilitating temporally efficient transfer (Bullmore and Sporns, 2009). Path length and clustering coefficients in the SNC and VTA dopaminergic networks were significantly different from random networks and random lattices of equivalent size and connection distribution. Although significantly different, these followed patterns found in random networks, suggesting 
that more complex topology may be present, such as small-worldness (Watts and Strogatz, 1998; Bassett and Bullmore, 2006).

We next sought to investigate SNC and VTA dopaminergic networks for small-world topology, which is characterized by high clustering, similar to random lattice clustering, and low path length, similar to random path length (Watts and Strogatz, 1998; Telesford et al., 2011). Importantly, small-world networks can confer processing efficiency, resiliency and synchronization (Watts and Strogatz, 1998; Albert et al., 2000; Lago-Fernández et al., 2000; Achard, 2006; Yu et al., 2008). SNC and VTA networks met the standard criteria of a smallworld coefficient $(\omega)$ between -0.5 and 0.5 (Telesford et al., 2011), suggesting that both regions exhibit this property robustly across time (Fig. $6 A-C$ ).

\section{SNC fragility and VTA resiliency in neuronal loss}

Although the SNC and VTA exhibit surprisingly similar and robust dynamic network properties, the literature suggests these regions to be differentially sensitive to neuronal loss (Sulzer, 2007; Mosharov et al., 2009; Lieberman et al., 2017). Furthermore, it remains unexplored whether loss of a single neuron significantly alters network properties. Therefore, we sought to investigate to what degree the functional network in each region is affected by the loss of a single neuron as determined by restructuring of network properties. To do so, we used simultaneous whole-cell patch clamp electrophysiology and calcium imaging to selectively hyperpolarize individual dopamine neurons to remove their contribution to the functional network activity (Fig. 7A). Network measurements were performed at two stages: (1) at baseline firing activity, while the neuron is patched, but no current is injected (Fig. $7 A$, note there is no change in the spontaneous firing of the patched neuron) and (2) during hyperpolarization, where a hyperpolarizing negative current is applied to suppress spontaneous firing activity (Fig. $7 A$, note the absence of firing activity during the hyperpolarization step).

Intriguingly, the networks retained many of their properties such as assortativity, which ascertains whether neurons preferentially connect to neurons with similar connection distributions, and modularity, which measures the degree to which the network forms subgroups (assortativity, $p=0.8127 \mathrm{SNC}, p=0.2418 \mathrm{VTA}$, modularity, $p=0.6604$ SNC, $p=0.1792$ VTA). Strikingly, we found that loss of an individual neuron in SNC increases clustering, suggesting that SNC neurons become more coordinated following neuronal loss to potentially overcome the loss and likely increases metabolic burden. Conversely, VTA networks exhibited significantly decreased clustering coefficients, which may provide resiliency to the region by preventing failures from cascading across the network.

\section{Dopamine transporter modulation regulates dopaminergic network properties}

Dopaminergic signaling is tightly regulated through dopamine transporter mediated reuptake. Previously we and others have shown that methamphetamine increases the DAT-dependent sodium current, intracellular calcium levels leading to increased firing frequency of dopamine neurons (Jones et al., 1999; Gnegy et al., 2004; Khoshbouei et al., 2004; Kahlig et al., 2005; Lin et al., 2016; Sambo et al., 2017). These data led us to the hypothesis that regulation of network properties may be under direct control of dopamine transporter activity. In order to test this hypothesis, we used methamphetamine to stimulate dopamine transporter activity. To determine specificity, these experiments were performed in the presence or absence of an antagonist cocktail that blocks the synaptic receptors D1, D2, GABA $\mathrm{GABA}_{\mathrm{B}}, \mathrm{AMPA}$, and NMDA (5 $\mu \mathrm{M}$ SCH-23390, $5 \mu \mathrm{M}$ sulpiride, $10 \mu \mathrm{m}$ SR-95531, $100 \mathrm{~nm}$ CGP-35348, $10 \mu \mathrm{M}$ CNQX, and $20 \mu \mathrm{M}$ MK-801, respectively) as well as a cocktail supplemented with a dopamine transporter inhibitor (nomifensine, $10 \mu \mathrm{M}$ ). Drug concentrations and duration of exposure $(6 \mathrm{~min})$ were chosen based on previous efficacious work (Lin et al., 2016; Sambo et al., 2017).This experimental paradigm identifies network properties that are specifically dependent on the activity of dopamine transporter, are dopamine receptor mediated, or occur independent of dopamine transporter or dopamine receptor activity. We found methamphetamine increased assortativity in both methamphetamine alone (drug vs baseline, $p=0.0152$, paired samples two-tailed $t$ test; Fig. $8 A$ ) and with antagonists (drug vs baseline, $p=0.0361$, paired samples twotailed $t$ test), but not in the presence of nomifensine blockade of dopamine transporter (drug vs baseline, $p=0.0503$, paired samples two-tailed $t$ test), although a similar trend is observed. A similar effect was observed for small-world coefficients (drug vs baseline, methamphetamine alone $p=0.0125$, 

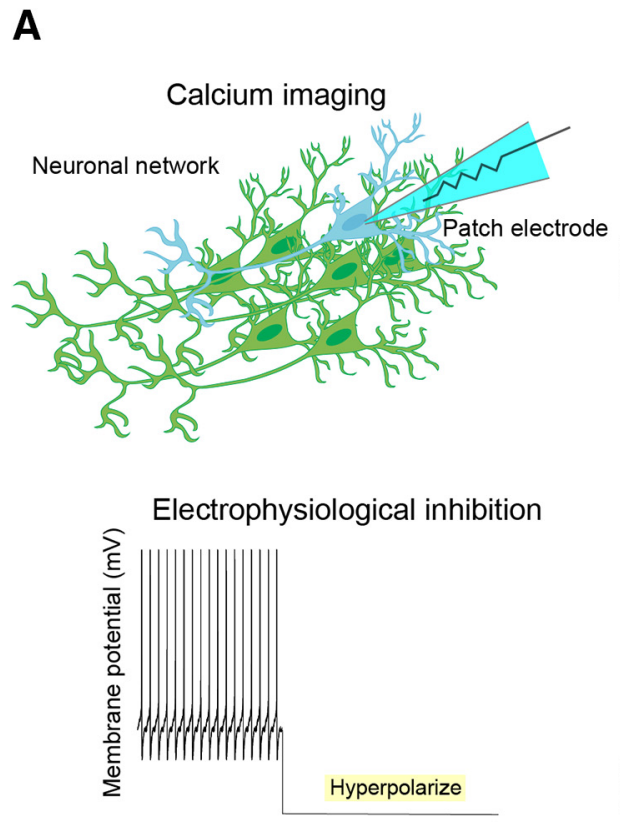

D
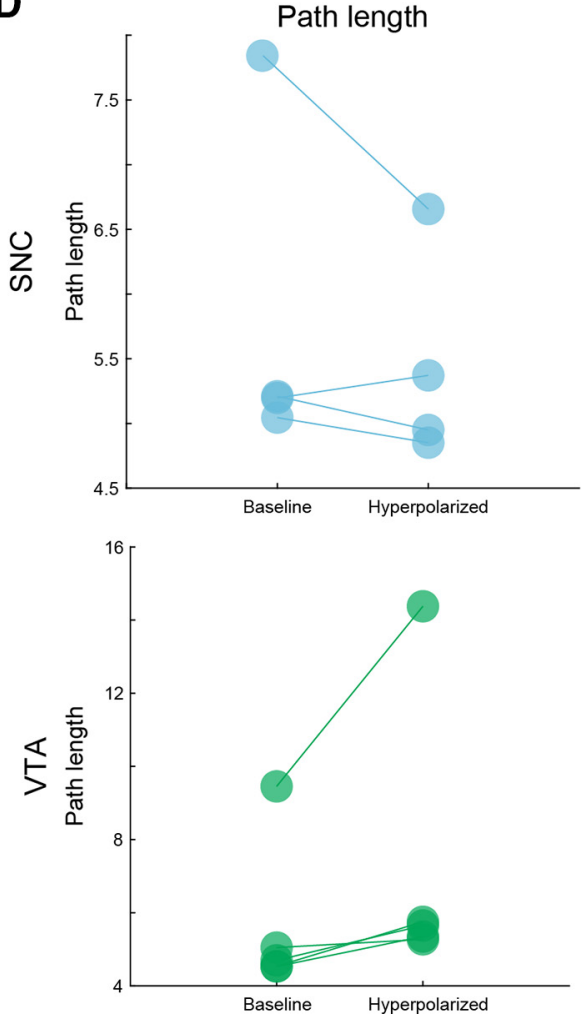
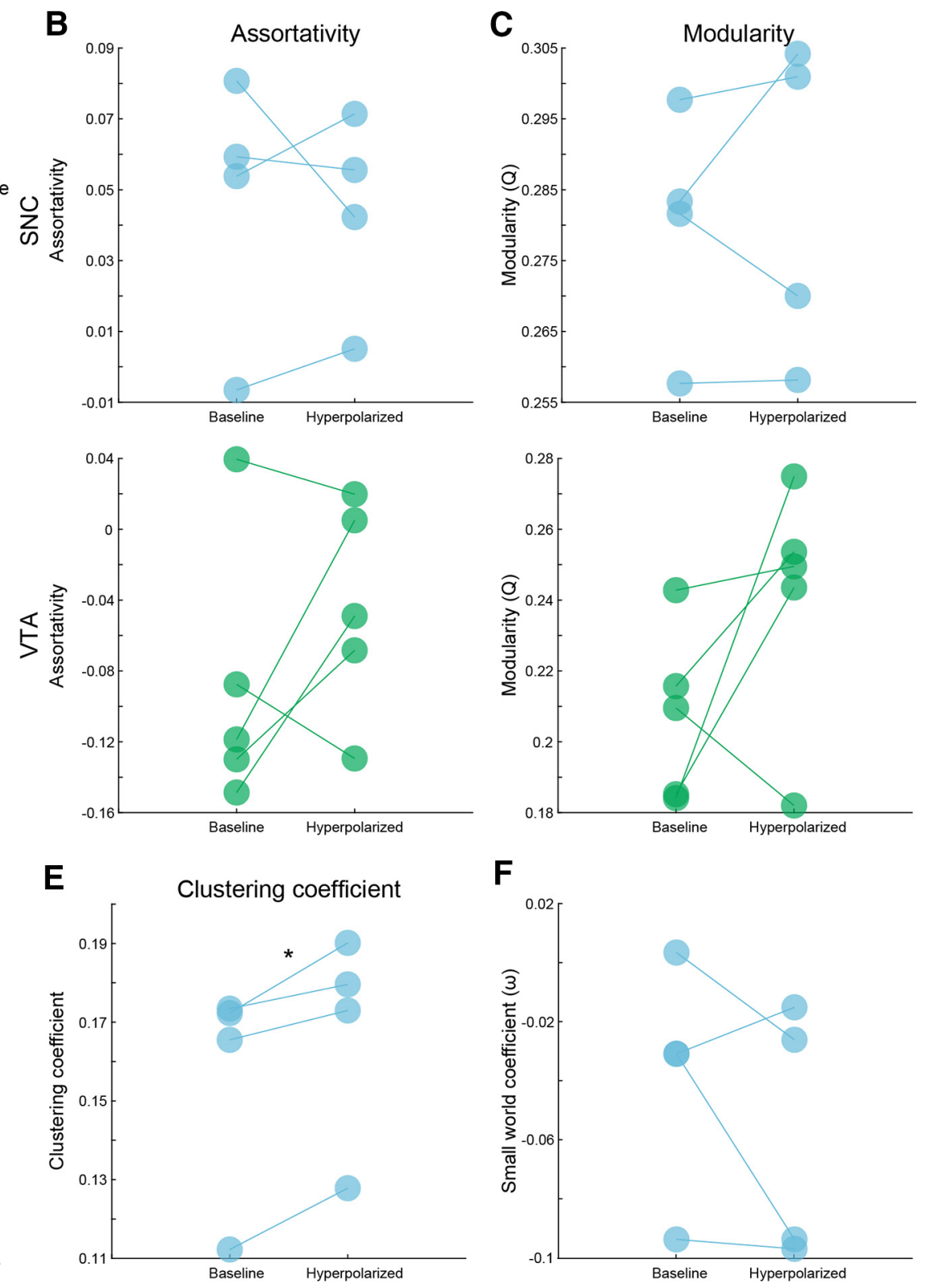

$\mathbf{F}$
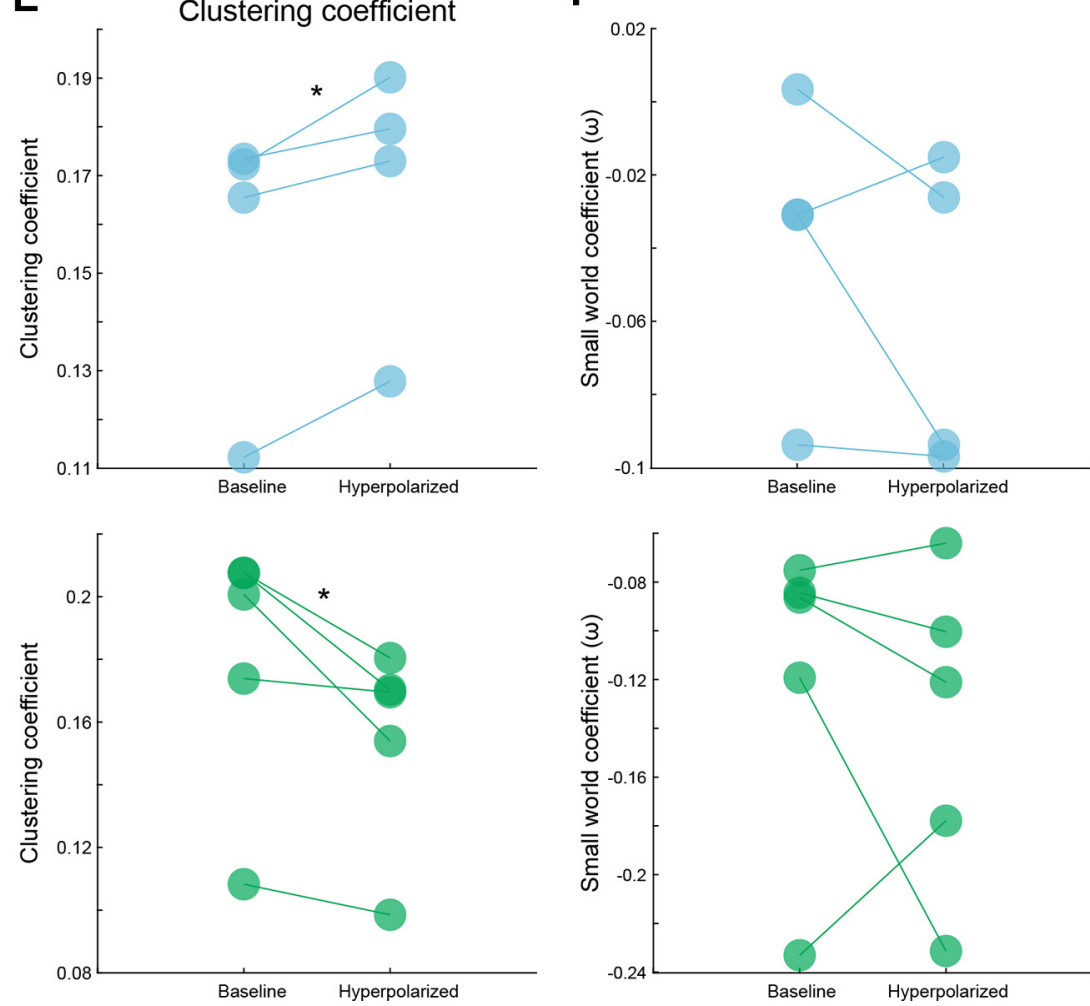

Figure 7. Suppression of individual neuronal activity increases network clustering in SNC but decreases network clustering in the VTA. A, top, Individual neurons within the field of view are patched sequentially with simultaneous calcium imaging. Middle, During the imaging paradigm, neurons are hyperpolarized to suppress their activity and eliminate contribution to the network. Bottom, Networks are analyzed with the neuron in the network (baseline) and while activity is suppressed (hyperpolarized). The hyperpolarized neurons were excluded from network measurements. Network assortativity $(\boldsymbol{B})$, modularity $(\boldsymbol{C})$, path length $(\boldsymbol{D})$, and small-world coefficient $(\boldsymbol{F})$ remained stable across the SNC and VTA when individual neurons were hyperpolarized (hyperpolarized vs baseline, assortativity, $p=0.8127$ SNC, $p=0.2418$ VTA, modularity, $p=0.6604$ SNC, $p=0.1792$ VTA, path length, $p=0.2947$ SNC, $p=0.1249$ VTA, small-world coefficient, $p=0.3264$ SNC, $p=0.5243$ VTA, paired samples two tailed $t$ test). Clustering coefficient (E) significantly differed in both regions (hyperpolarized vs baseline, $p=0.0271$ SNC, 0.0353 VTA, twotailed paired samples $t$ test), but were opposite in valence; $n=4-5$ networks per group; ${ }^{*} p<0.05$. 
A

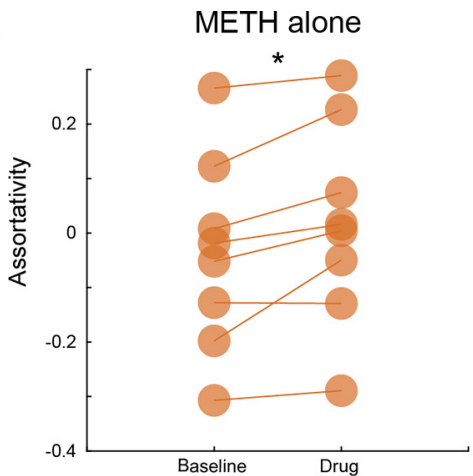

B

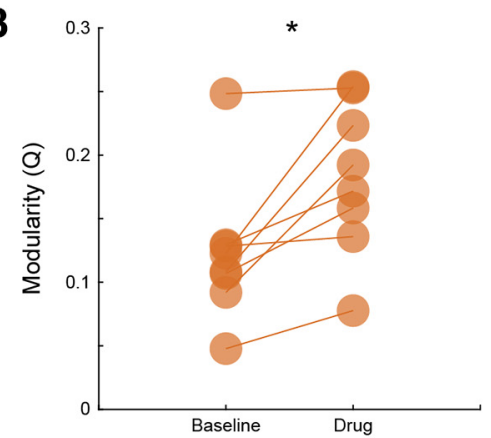

C

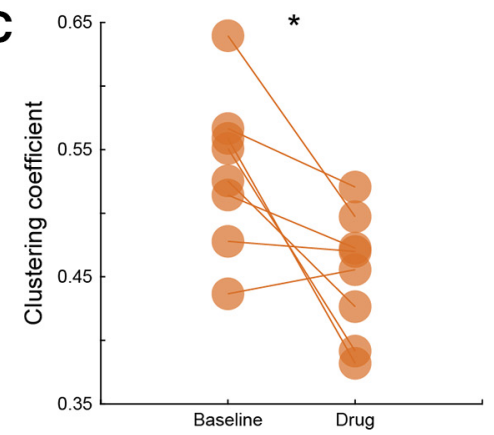

D

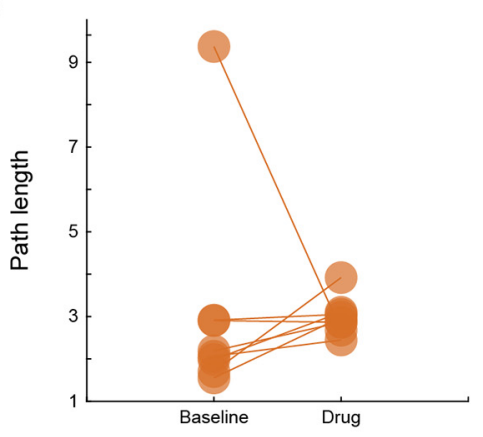

E

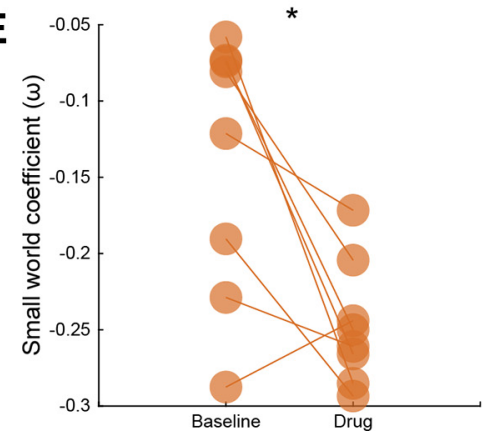

METH with antagonists
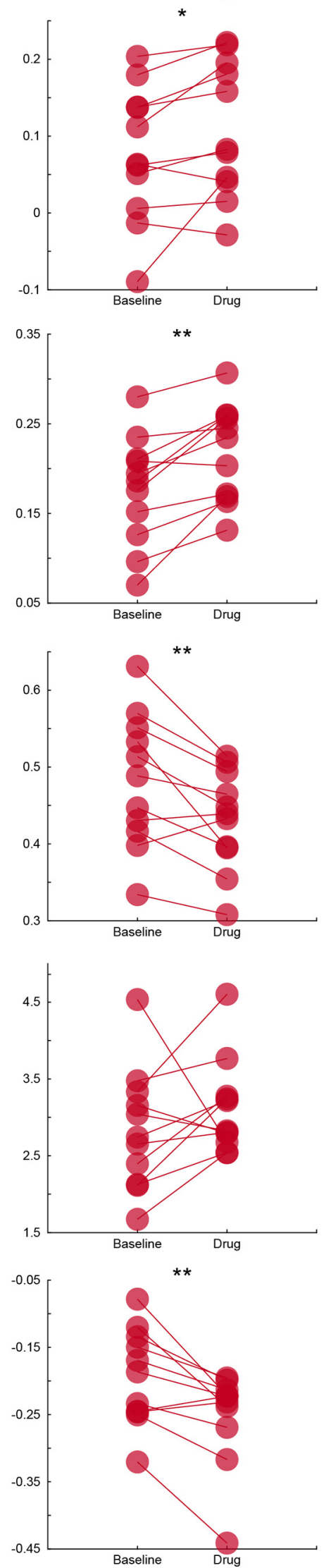

METH, nomifensine, antagonists
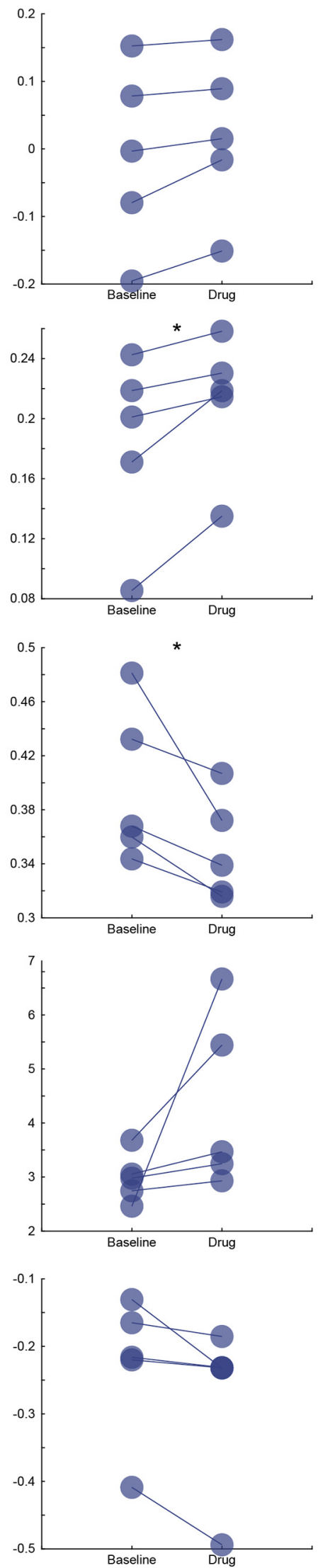

Figure 8. Dopamine transporter modulation regulates dopaminergic network properties. $A$, Dopaminergic networks stimulated with methamphetamine exhibit increased assortativity whether methamphetamine alone or with antagonist cocktail blocking: D1, D2, GABA $A_{A}, G A B A_{B}$, NMDA, AMPA receptors is applied (methamphetamine alone $p=0.0152$, methamphetamine with antagonists $p=0.0361, p=0.0503$ methamphetamine with nomifensine and antagonists, two-tailed paired samples $t$ test). $\boldsymbol{B}$, Network modularity increased across all conditions 
methamphetamine with antagonist cocktail $p=0.0046$, methamphetamine, nomifensine, and antagonists $p=0.0713$, paired samples two-tailed $t$ test; Fig. $8 E$ ). Since methamphetamine decreases dopamine uptake (Bennett et al., 1998; Sulzer et al., 2005), increases dopamine efflux (Stephans and Yamamoto, 1995; Kahlig et al., 2005; Fog et al., 2006), and increases firing activity of dopamine neurons (Branch and Beckstead, 2012; Saha et al., 2014; Lin et al., 2016), it is not surprising that methamphetamine exposure increased modularity and reduced network clustering across all conditions (drug vs baseline, modularity methamphetamine alone $p=0.0104$, methamphetamine with antagonist cocktail $p=0.0012$, methamphetamine, nomifensine, and antagonists $p=0.0323$, clustering coefficient, methamphetamine alone $p=0.0159$, methamphetamine with antagonist cocktail $p=0.0068$, methamphetamine, nomifensine, and antagonists $p=0.0488$, paired samples two-tailed $t$ test; Fig. $8 B, C$ ), while path length remained consistent throughout (methamphetamine alone $p=0.9238$, methamphetamine with antagonist cocktail $p=0.3215$, methamphetamine, nomifensine, and antagonists $p=0.1489$, paired samples two-tailed $t$ test; Fig. $8 D$ ). These results collectively suggest that modulation of dopamine transporter activity can regulate dopaminergic network properties.

\section{Technical and conceptual limitations of this study}

In the current study, we leveraged genetic restriction of GCaMP and complex network analysis to determine the intrinsic properties of dopaminergic networks of the SNC and VTA. Although this study is the first to our knowledge to examine dopaminergic networks in this manner, the methods employed also impose technical and conceptual limitations in the study. Specifically, the utilization of slices in the coronal plane undoubtedly sever many physical connections and projections between neurons. Furthermore, the $40 \times$ objective and 1-photon imaging used limits the observable region, and correspondingly the number of neurons, than can be achieved with a $20 \times$ objective (Cossart et al., 2003; Bonifazi et al., 2009; Pachitariu et al., 2017). We have formed our interpretations with these concerns (van Wijk et al., 2010; Miele et al., 2019) in mind, and future studies will overcome these limitations as dopaminergic networks are further examined in multiple planes, as well as the potential for in vivo implementations that have recently begun to be explored in these regions (da Silva et al., 2018; Fernandes et al., 2020a).

\section{Discussion}

Dopaminergic neurons and by proxy their regional networks exhibit a rich diversity of neurochemical and electrophysiological properties (Margolis et al., 2006; Fu et al., 2012). The focus of the field often relies on results from individually recorded neurons (Ingram et al., 2002; Margolis et al., 2006; Puopolo et al.,

$\leftarrow$

(methamphetamine alone $p=0.0104$, methamphetamine with antagonists $p=0.0012$, methamphetamine with nomifensine and antagonists $p=0.0323$, two-tailed paired samples $t$ test). C, Clustering coefficients of dopaminergic networks significantly decreased across all conditions (methamphetamine alone $p=0.0159$, methamphetamine with antagonists $p=0.0068$, methamphetamine with nomifensine and antagonists $=0.0488$, two-tailed paired samples $t$ test). $\boldsymbol{D}$, Path length remained the same across all stimulation conditions (methamphetamine alone $p=0.9238$, methamphetamine with antagonists $p=0.3215$, methamphetamine with nomifensine and antagonists $p=0.1489$, two-tailed paired samples $t$ test). $E$, Methamphetamine stimulation promoted lattice-like small-worlds in dopaminergic networks, but not when dopamine transporter is occluded (methamphetamine alone $p=0.0125$, methamphetamine with antagonists $p=0.0046$, methamphetamine with nomifensine and antagonists $p=0.0713$, two-tailed paired samples $t$ test); $n=5-11$ networks per group; ${ }^{*} p<0.05,{ }^{* *} p<0.01$.
2007; Lin et al., 2016, 2020), or distinctions derived from immunohistochemistry (Fu et al., 2012) or transcriptomics (Poulin et al., 2014; Tiklová et al., 2019) that cannot capture whether the activity in one neuron influence the activity of local neurons and ultimately the output of dopaminergic network. To address these limitations, we used simultaneous highspeed calcium imaging and electrophysiology to isolate interregional differences in interactions of spontaneously active dopaminergic neurons of neighboring dopaminergic nuclei, the SNC and VTA at baseline and following exposure to methamphetamine known to increase dopamine transmission via multiple mechanisms.

Our results show for the first time that the dopaminergic networks of the SN (SNC) and VTA exhibit stable and rich dynamic network properties. We identified increased prevalence of network hubs within the VTA compared with the SNC, differential responses to loss of a single neuron, and modulation through dopamine transporter dependent and independent mechanisms.

Functional networks emerge from local interactions between neurons (Gutierrez and Marder, 2014; Schroeter et al., 2015; Palacios et al., 2019). In dopaminergic networks, dopamine neurons have the machinery to directly interact through both synaptic and non-synaptic signaling (Beckstead and Williams, 2007; Aversa et al., 2018; Miller et al., 2019). However, these studies do not account for dopaminergic network properties and dynamics, and how they differ by brain region. Identification of regional differences offer greater understanding of differential sensitivity of dopaminergic regions, such as loss of SNC dopamine neurons in Parkinson's disease (Sulzer, 2007; Mosharov et al., 2009; Vaillancourt et al., 2009). Within the subsets of each regional area sampled, we found an overrepresentation of hub-like neurons in VTA dopaminergic networks than in the adjacent SNC. Therefore, the event of loss of a hub neuron in the SNC would have a greater detriment to behavioral outputs that are circumscribed to the SNC. Finally, although the observed regions represent a small fraction of the total area of the region, which is the general limitation of live cell imaging in the randomly selected regions in the SN and VTA, our computational analyses still revealed non-trivial differences in network topology between regions. Future work will determine the degree to which this is consistent across the three-dimensional axes (rostral-caudal/ medial-lateral) of the SNC and VTA.

The observed differences in basal network properties led us to test the hypothesis that neuronal loss should differentially affect these two regions. Selective removal of neurons from functional networks through electrophysiological suppression resulted in surprisingly increased clustering of the SNC with decreased clustering in the VTA (Fig. 7E). While high clustering, a higher order measure of connectivity (MacLean et al., 2005; Chambers and MacLean, 2016), is associated with network robustness (Bullmore and Sporns, 2009), increases in clustering suggest that SNC networks respond to neuronal loss by increasing functional segregation (Rubinov and Sporns, 2010) to potentially maintain network output, while the VTA responds by becoming more dispersive. Higher clustering in the SNC suggests an increase of neuronal resources that would maintain network output despite the reported lower average firing rates which have been observed previously (Janezic et al., 2013). Increasing resources may create metabolic stressors that may predispose further neuronal dysfunction and consequently network failure (Thompson, 2004; Ekstrand and Galter, 2009; Lieberman et al., 2017). Thus, the network attempting to maintain synchrony by temporally aligning may then contribute to dysfunction of other nuclei within the basal ganglia (Brown, 
2003; Burkhardt et al., 2009). Conversely, the response found within the VTA would suggest robustness resilience to cascades of energy usage or pathogens (Rubinov and Sporns, 2010; Cai et al., 2020). However, loss or dysfunction of VTA dopamine neurons also occurs in Parkinson's disease and experience cognitive deficits associated with VTA dopamine neurons (Alberico et al., 2015). A VTA lesion study found unrefined executive functioning (Pioli et al., 2008), as was also found in a case report of a patient exhibiting VTA damage (Adair et al., 1996). Furthermore, cortical areas innervated by the VTA exhibit hypodopaminergic states in schizophrenia (da Silva Alves et al., 2008; Pogarell et al., 2012) and lower clustering coefficients (Lynall et al., 2010). Therefore, given enough dispersion of VTA dopamine networks, the outcome of loss of higher order synchrony and functional specialization may underlie these symptoms.

Beyond single neurons, dopaminergic networks feature volume transmission and therefore can interact with both proximal and distal targets (Rice, 2000; Rice and Patel, 2015). Dopamine transporter regulates the degree to which dopamine diffusion can occur through the reuptake of dopamine and ending dopamine signaling (Jones et al., 1999; Cragg et al., 2001). Furthermore, influx of dopamine or methamphetamine through the transporter produces depolarizing currents that alter the firing properties of these neurons (Ingram et al., 2002; Lin et al., 2016; Aversa et al., 2018), which both transporter activity and firing rate are critical to dopaminergic synchronization (Dreyer and Hounsgaard, 2013; Beeler and Dreyer, 2019). Disruption of dopamine transporter function would therefore likely cascade across the network, disrupting network topology. Our data support the interpretation that network structure is under tight control by dopamine transporter, as all but one metric (path length) significantly altered through manipulation of dopamine transporter, and these effects persisted under blockade of many of the receptor types found within the ventral midbrain, but not when the transporter was blocked (Fig. 8). Therefore, dopamine transporter can act as a master regulator of dopaminergic networks through regulation of dopaminergic signaling. Additionally, this provides a direct pharmacological target that may be able to revert dopaminergic networks with dysfunctional properties, such as those found in Parkinson's disease (Fischer et al., 2019) and addiction (Tomasi et al., 2009; Ding et al., 2013; Orsini et al., 2018). However, as the experiments were performed in a cocktail of antagonists, future experiments will be necessary to provide more nuanced interpretations to the regulation of dopaminergic networks through simultaneous polypharmacy approaches of multiple targets that have been shown to alter network synchrony (Li et al., 2011). Importantly, while acute midbrain slices provide regional investigations into the regulatory mechanisms within each region, they do not account for active afferent input during behavioral state-dependent changes observed in freely behaving animals (Arbuthnott and Wickens, 2007; Kim et al., 2012; Lohani et al., 2019). Therefore, future experiments will require in vivo monitoring of network activity to determine whether and how dopaminergic networks of the SNC and VTA shift their topology to produce or respond to statedependent changes.

These results expand and complement the current body of literature from the firing rates of individual neurons and synchrony to networks of interacting dopaminergic neurons whose remarkable heterogeneity give rise to exquisite regional differences, both in sensitivity and resiliency. Therefore, our approach provides a foundation for future experiments into the functional structure of dopaminergic networks and how changes in functional structure underlie behavior and disease.

\section{References}

Achard S (2006) A resilient, low-frequency, small-world human brain functional network with highly connected association cortical hubs. J Neurosci 26:63-72.

Adair JC, Williamson DJ, Schwartz RL, Heilman KM (1996) Ventral tegmental area injury and frontal lobe disorder. Neurology 46:842843.

Alberico SL, Cassell MD, Narayanan NS (2015) The vulnerable ventral tegmental area in Parkinson's disease. Basal Ganglia 5:51-55.

Albert R, Jeong H, Barabasi AL (2000) Error and attack tolerance of complex networks. Nature 406:378-382.

Arbuthnott GW, Wickens J (2007) Space, time and dopamine. Trends Neurosci 30:62-69.

Aversa D, Martini A, Guatteo E, Pisani A, Mercuri NB, Berretta N (2018) Reversal of dopamine-mediated firing inhibition through activation of the dopamine transporter in substantia nigra pars compacta neurons. Br J Pharmacol 175:3534-3547.

Bassett DS, Bullmore E (2006) Small-world brain networks. Neuroscientist 12:512-523.

Bassett DS, Sporns O (2017) Network neuroscience. Nat Neurosci 20:353364.

Bastian M, Heymann S, Jacomy M (2009) Gephi: an open source software for exploring and manipulating networks. Icwsm 8:361-362.

Bean BP (2007) The action potential in mammalian central neurons. Nat Rev Neurosci 8:451-465.

Beckstead MJ, Williams JT (2007) Long-term depression of a dopamine IPSC. J Neurosci 27:2074-2080.

Beckstead MJ, Grandy DK, Wickman K, Williams JT (2004) Vesicular dopamine release elicits an inhibitory postsynaptic current in midbrain dopamine neurons. Neuron 42:939-946.

Beeler JA, Mourra D (2018) To do or not to do: dopamine, affordability and the economics of opportunity. Front Integr Neurosci 12:6.

Beeler JA, Dreyer JK (2019) Synchronicity: the role of midbrain dopamine in whole brain coordination. eNeuro 6:ENEURO.0345-18.2019.

Bennett BA, Hollingsworth CK, Martin RS, Harp JJ (1998) Methamphetamineinduced alterations in dopamine transporter function. Brain Res 782:219227.

Benoit-Marand M, Borrelli E, Gonon F (2001) Inhibition of dopamine release via presynaptic D2 receptors: time course and functional characteristics in vivo. J Neurosci 21:9134-9141.

Berke JD (2018) What does dopamine mean? Nat Neurosci 21:787-793.

Boger HA, Middaugh LD, Zaman V, Hoffer B, Granholm AC (2008) Differential effects of the dopamine neurotoxin MPTP in animals with a partial deletion of the GDNF receptor, GFR alphal, gene. Brain Res 1241:18-28.

Bonifazi P, Goldin M, Picardo MA, Jorquera I, Cattani A, Bianconi G, Represa A, Ben-Ari Y, Cossart R (2009) GABAergic hub neurons orchestrate synchrony in developing hippocampal networks. Science 326:14191424.

Borisovska M, Bensen AL, Chong G, Westbrook GL (2013) Distinct modes of dopamine and GABA release in a dual transmitter neuron. J Neurosci 33:1790-1796.

Braak H, Braak E (2000) Pathoanatomy of Parkinson's disease. J Neurol 247: II3-10.

Branch SY, Beckstead MJ (2012) Methamphetamine produces bidirectional, concentration-dependent effects on dopamine neuron excitability and dopamine-mediated synaptic currents. J Neurophysiol 108:802-809.

Branch SY, Sharma R, Beckstead MJ (2014) Aging decreases L-type calcium channel currents and pacemaker firing fidelity in substantia nigra dopamine neurons. J Neurosci 34:9310-9318.

Brown MTC, Henny P, Bolam JP, Magill PJ (2009) Activity of neurochemically heterogeneous dopaminergic neurons in the substantia nigra during spontaneous and driven changes in brain state. J Neurosci 29:2915-2925. 
Brown P (2003) Oscillatory nature of human basal ganglia activity: relationship to the pathophysiology of Parkinson's disease. Mov Disord 18:357363.

Bullmore ET, Sporns O (2009) Complex brain networks: graph theoretical analysis of structural and functional systems. Nat Rev Neurosci 10:186198.

Burkhardt JM, Jin X, Costa RM (2009) Dissociable effects of dopamine on neuronal firing rate and synchrony in the dorsal striatum. Front Integr Neurosci 3:28.

Cai L, Wei X, Liu J, Zhu L, Wang J, Deng B, Yu H, Wang R (2020) Functional integration and segregation in multiplex brain networks for Alzheimer's. Front Neurosci 14:51.

Chambers B, MacLean JN (2016) Higher-order synaptic interactions coordinate dynamics in recurrent networks. PLoS Comput Biol 12: e1005078.

Chang L, Wang GJ, Volkow ND, Ernst T, Telang F, Logan J, Fowler JS (2008) Decreased brain dopamine transporters are related to cognitive deficits in HIV patients with or without cocaine abuse. Neuroimage 42:869-878.

Chen TW, Wardill TJ, Sun Y, Pulver SR, Renninger SL, Baohan A, Schreiter ER, Kerr RA, Orger MB, Jayaraman V, Looger LL, Svoboda K, Kim DS (2013) Ultrasensitive fluorescent proteins for imaging neuronal activity. Nature 499:295-300.

Cork SC, Richards JE, Holt MK, Gribble FM, Reimann F, Trapp S (2015) Distribution and characterisation of Glucagon-like peptide-1 receptor expressing cells in the mouse brain. Mol Metab 4:718-731.

Cossart R, Aronov D, Yuste R (2003) Attractor dynamics of network UP states in the neocortex. Nature 423:283-288.

Cragg SJ, Rice ME (2004) DAncing past the DAT at a DA synapse. Trends Neurosci 27:270-277.

Cragg SJ, Nicholson C, Kume-Kick J, Tao L, Rice ME (2001) Dopaminemediated volume transmission in midbrain is regulated by distinct extracellular geometry and uptake. J Neurophysiol 85:1761-1771.

Cruz HG, Ivanova T, Lunn M-L, Stoffel M, Slesinger PA, Lüscher C (2004) Bi-directional effects of GABAB receptor agonists on the mesolimbic dopamine system. Nat Neurosci 7:153-159.

da Silva JA, Tecuapetla F, Paixão V, Costa RM (2018) Dopamine neuron activity before action initiation gates and invigorates future movements. Nature 554:244-248.

da Silva Alves F, Figee M, van Amelsvoort T, Veltman D, de Haan L (2008) The revised dopamine hypothesis of schizophrenia: evidence from pharmacological MRI studies with atypical antipsychotic medication. Psychopharmacol Bull 41:121-132.

Ding WN, Sun JH, Sun YW, Zhou Y, Li L, Xu JR, Du YS (2013) Altered default network resting-state functional connectivity in adolescents with Internet gaming addiction. PLoS One 8:e59902.

Dreyer JK, Hounsgaard J (2013) Mathematical model of dopamine autoreceptors and uptake inhibitors and their influence on tonic and phasic dopamine signaling. J Neurophysiol 109:171-182.

Dubbs A, Guevara J, Yuste R (2016) moco: fast motion correction for calcium imaging. Front Neuroinform 10:6.

Ekstrand MI, Galter D (2009) The MitoPark mouse - an animal model of Parkinson's disease with impaired respiratory chain function in dopamine neurons. Parkinsonism Relat Disord 15:S185-S188.

Engelhard B, Finkelstein J, Cox J, Fleming W, Jang HJ, Ornelas S, Koay SA, Thiberge SY, Daw ND, Tank DW, Witten IB (2019) Specialized coding of sensory, motor and cognitive variables in VTA dopamine neurons. Nature 570:509-513.

Evans RC, Zhu M, Khaliq ZM (2017) Dopamine inhibition differentially controls excitability of substantia nigra dopamine neuron subpopulations through T-type calcium channels. J Neurosci 37:3704-3720.

Fernandes AB, Alves da Silva J, Almeida J, Cui G, Gerfen CR, Costa RM, Oliveira-Maia AJ (2020a) Postingestive modulation of food seeking depends on vagus-mediated dopamine neuron activity. Neuron 106:778788.e6.

Fernandes HJR, Patikas N, Foskolou S, Field SF, Park J-E, Byrne ML, Bassett AR, Metzakopian E (2020b) Single-cell transcriptomics of Parkinson's disease human in vitro models reveals dopamine neuron-specific stress responses. Cell Rep 33:108263.

Figlewicz DP, Evans SB, Murphy J, Hoen M, Baskin DG (2003) Expression of receptors for insulin and leptin in the ventral tegmental area/substantia nigra (VTA/SN) of the rat. Brain Res 964:107-115.
Fischer P, Pogosyan A, Green AL, Aziz TZ, Hyam J, Foltynie T, Limousin P, Zrinzo L, Samuel M, Ashkan K, Da Lio M, De Cecco M, Fornaser A, Brown P, Tan H (2019) Beta synchrony in the cortico-basal ganglia network during regulation of force control on and off dopamine. Neurobiol Dis 127:253-263.

Fog JU, Khoshbouei H, Holy M, Owens WA, Vaegter CB, Sen N, Nikandrova Y, Bowton E, McMahon DG, Colbran RJ, Daws LC, Sitte HH, Javitch JA, Galli A, Gether U (2006) Calmodulin kinase II interacts with the dopamine transporter $\mathrm{C}$ terminus to regulate amphetamineinduced reverse transport. Neuron 51:417-429.

Foster JG, Foster DV, Grassberger P, Paczuski M (2010) Edge direction and the structure of networks. Proc Natl Acad Sci USA 107:1081510820.

Fu YH, Yuan Y, Halliday G, Rusznák KZ, Watson C, Paxinos G (2012) A cytoarchitectonic and chemoarchitectonic analysis of the dopamine cell groups in the substantia nigra, ventral tegmental area, and retrorubral field in the mouse. Brain Struct Funct 217:591-612.

Gallos LK, Song C, Makse HA (2008) Scaling of degree correlations and its influence on diffusion in scale-free networks. Phys Rev Lett 100:248701.

Gantz SC, Bunzow JR, Williams JT (2013) Spontaneous inhibitory synaptic currents mediated by a G protein-coupled receptor. Neuron 78:807-812.

Gao J, Barzel B, Barabási AL (2016) Universal resilience patterns in complex networks. Nature 530:307-312.

Garrison KA, Scheinost D, Finn ES, Shen X, Constable RT (2015) The (in) stability of functional brain network measures across thresholds. Neuroimage 118:651-661.

Gentet LJ, Williams SR (2007) Dopamine gates action potential backpropagation in midbrain dopaminergic neurons. J Neurosci 27:1892-1901.

Giguère N, Delignat-Lavaud B, Herborg F, Voisin A, Li Y, Jacquemet V, Anand-Srivastava M, Gether U, Giros B, Trudeau LÉ (2019) Increased vulnerability of nigral dopamine neurons after expansion of their axonal arborization size through D2 dopamine receptor conditional knockout. PLoS Genet 15:e1008352.

Gnegy ME, Khoshbouei H, Berg KA, Javitch JA, Clarke WP, Zhang M, Galli A (2004) Intracellular $\mathrm{Ca} 2+$ regulates amphetamine-induced dopamine efflux and currents mediated by the human dopamine transporter. Mol Pharmacol 66:137-143.

Goodwin JS, Larson GA, Swant J, Sen N, Javitch JA, Zahniser NR, De Felice LJ, Khoshbouei H (2009) Amphetamine and methamphetamine differentially affect dopamine transporters in vitro and in vivo. J Biol Chem 284:2978-2989.

Grace AA, Bunney BS (1983) Intracellular and extracellular electrophysiology of nigral dopaminergic neurons-1. Identification and characterization. Neuroscience 10:301-315.

Grieder TE, Herman MA, Contet C, Tan LA, Vargas-Perez H, Cohen A, Chwalek M, Maal-Bared G, Freiling J, Schlosburg JE, Clarke L, Crawford E, Koebel P, Repunte-Canonigo V, Sanna PP, Tapper AR, Roberto M, Kieffer BL, Sawchenko PE, Koob GF, et al. (2014) VTA CRF neurons mediate the aversive effects of nicotine withdrawal and promote intake escalation. Nat Neurosci 17:17511758

Gutierrez GJ, Marder E (2014) Modulation of a single neuron has state-dependent actions on circuit dynamics. eNeuro 1: ENEURO.0009-14.2014.

Hnasko TS, Chuhma N, Zhang H, Goh GY, Sulzer D, Palmiter RD, Rayport S, Edwards RH (2010) Vesicular glutamate transport promotes dopamine storage and glutamate corelease in vivo. Neuron 65:643-656.

Howell RD, Dominguez-Lopez S, Ocañas SR, Freeman WM, Beckstead MJ (2020) Female mice are resilient to age-related decline of substantia nigra dopamine neuron firing parameters. Neurobiol Aging 95:195-204.

Ingram SL, Prasad BM, Amara SG (2002) Dopamine transporter-mediated conductances increase excitability of midbrain dopamine neurons. Nat Neurosci 5:971-978.

Janezic S, Threlfell S, Dodson PD, Dowie MJ, Taylor TN, Potgieter D, Parkkinen L, Senior SL, Anwar S, Ryan B, Deltheil T, Kosillo P, Cioroch M, Wagner K, Ansorge O, Bannerman DM, Bolam JP, Magill PJ, Cragg SJ, Wade-Martins R (2013) Deficits in dopaminergic transmission precede neuron loss and dysfunction in a new Parkinson model. Proc Natl Acad Sci USA 110:E4016-E4025. 
Jayaraman A, Nishimori T, Dobner P, Uhl GR (1990) Cholecystokinin and neurotensin mRNAs are differentially expressed in subnuclei of the ventral tegmental area. J Comp Neurol 296:291-302.

Jones SR, Joseph JD, Barak LS, Caron MG, Wightman RM (1999) Dopamine neuronal transport kinetics and effects of amphetamine. J Neurochem 73:2406-2414

Kahlig KM, Binda F, Khoshbouei H, Blakely RD, McMahon DG, Javitch JA, Galli A (2005) Amphetamine induces dopamine efflux through a dopamine transporter channel. Proc Natl Acad Sci USA 102:3495-3500.

Kahlig KM, Lute BJ, Wei Y, Loland CJ, Gether U, Javitch JA, Galli A (2006) Regulation of dopamine transporter trafficking by intracellular amphetamine. Mol Pharmacol 70:542-548.

Khoshbouei H, Sen N, Guptaroy B, Johnson L, Lund D, Gnegy ME, Galli A, Javitch JA (2004) N-terminal phosphorylation of the dopamine transporter is required for amphetamine-induced efflux. PLoS Biol 2:E78.

Kim Y, Wood J, Moghaddam B (2012) Coordinated activity of ventral tegmental neurons adapts to appetitive and aversive learning. PLoS One 7: e29766.

Knowlton C, Kutterer S, Roeper J, Canavier CC (2018) Calcium dynamics control K-ATP channel-mediated bursting in substantia nigra dopamine neurons: a combined experimental and modeling study. J Neurophysiol 119:84-95.

Korotkova TM, Sergeeva OA, Eriksson KS, Haas HL, Brown RE (2003) Excitation of ventral tegmental area dopaminergic and nondopaminergic neurons by orexins/hypocretins. J Neurosci 23:7-11.

Krasnova IN, Ladenheim B, Hodges AB, Volkow ND, Cadet JL (2011) Chronic methamphetamine administration causes differential regulation of transcription factors in the rat midbrain. PLoS One 6: e19179.

Kremer Y, Flakowski J, Rohner C, Lüscher C (2020) Context-dependent multiplexing by individual VTA dopamine neurons. J Neurosci 40:74897509.

La Manno G, Gyllborg D, Codeluppi S, Nishimura K, Salto C, Zeisel A, Borm LE, Stott SRW, Toledo EM, Villaescusa JC, Lönnerberg P, Ryge J, Barker RA, Arenas E, Linnarsson S (2016) Molecular diversity of midbrain development in mouse, human, and stem cells. Cell 167:566-580.e19.

Lago-Fernández LF, Huerta R, Corbacho F, Sigüenza JA (2000) Fast response and temporal coherent oscillations in small-world networks. Phys Rev Lett 84:2758-2761.

Langer N, Pedroni A, Jäncke L (2013) The problem of thresholding in smallworld network analysis. PLoS One 8:e53199.

Lerner TN, Shilyansky C, Davidson TJ, Evans KE, Beier KT, Zalocusky KA, Crow AK, Malenka RC, Luo L, Tomer R, Deisseroth K (2015) Intactbrain analyses reveal distinct information carried by $\mathrm{SNc}$ dopamine subcircuits. Cell 162:635-647.

Li W, Doyon WM, Dani JA (2011) Acute in vivo nicotine administration enhances synchrony among dopamine neurons. Biochem Pharmacol 82:977-983.

Liang CL, Sinton CM, German DC (1996) Midbrain dopaminergic neurons in the mouse: co-localization with calbindin- $\mathrm{D}(28 \mathrm{~K})$ and calretinin. Neuroscience 75:523-533.

Lieberman OJ, Choi SJ, Kanter E, Saverchenko A, Frier MD, Fiore GM, Wu M, Kondapalli J, Zampese E, Surmeier DJ, Sulzer D, Mosharov EV (2017) $\alpha$-Synuclein-dependent calcium entry underlies differential sensitivity of cultured SN and VTA dopaminergic neurons to a Parkinsonian neurotoxin. eNeuro 4:ENEURO.0167-17.2017.

Lim S, Radicchi F, van den Heuvel MP, Sporns O (2019) Discordant attributes of structural and functional brain connectivity in a two-layer multiplex network. Sci Rep 9:2885.

Lin M, Sambo D, Khoshbouei H (2016) Methamphetamine regulation of firing activity of dopamine neurons. J Neurosci 36:10376-10391.

Lin M, Colon-Perez LM, Sambo DO, Miller DR, Lebowitz JJ, JimenezRondan F, Cousins RJ, Horenstein N, Aydemir TB, Febo M, Khoshbouei $\mathrm{H}$ (2020) Mechanism of manganese dysregulation of dopamine neuronal activity. J Neurosci 40:5871-5891.

Lindgren N, Xu ZQD, Herrera-Marschitz M, Haycock J, Hökfelt T, Fisone G (2001) Dopamine $\mathrm{D}(2)$ receptors regulate tyrosine hydroxylase activity and phosphorylation at Ser40 in rat striatum. Eur J Neurosci 13:773-780.
Lohani S, Martig AK, Deisseroth K, Witten IB, Moghaddam B (2019) Dopamine modulation of prefrontal cortex activity is manifold and operates at multiple temporal and spatial scales. Cell Rep 27:99114.e6.

Lurie DJ, Kessler D, Bassett DS, Betzel RF, Breakspear M, Kheilholz S, Kucyi A, Liégeois R, Lindquist MA, McIntosh AR, Poldrack RA, Shine JM, Thompson WH, Bielczyk NZ, Douw L, Kraft D, Miller RL, Muthuraman M, Pasquini L, Razi A, et al. (2020) Questions and controversies in the study of time-varying functional connectivity in resting fMRI. Netw Neurosci 4:30-69.

Lüscher C (2016) The emergence of a circuit model for addiction. Annu Rev Neurosci 39:257-276.

Lynall M-E, Bassett D, Kerwin R, McKenna P, Kitzbichler M, Muller U, Bullmore E (2010) Functional connectivity and brain networks in schizophrenia. J Neurosci 30:9477-9487.

MacLean JN, Watson BO, Aaron GB, Yuste R (2005) Internal dynamics determine the cortical response to thalamic stimulation. Neuron 48:811823.

Margolis EB, Lock H, Hjelmstad GO, Fields HL (2006) The ventral tegmental area revisited: is there an electrophysiological marker for dopaminergic neurons? J Physiol 577:907-924.

Martin CA, Myers KM, Chen A, Martin NT, Barajas A, Schweizer FE, Krantz DE (2016) Ziram, a pesticide associated with increased risk for Parkinson's disease, differentially affects the presynaptic function of aminergic and glutamatergic nerve terminals at the Drosophila neuromuscular junction. Exp Neurol 275:232-241.

Maslov S, Sneppen K (2002) Specificity and stability in topology of protein networks. Science 296:910-913.

Mattammal MB, Haring JH, Chung HD, Raghu G, Strong R (1995) An endogenous dopaminergic neurotoxin: implication for Parkinson's disease. Neurodegeneration 4:271-281.

Miele V, Matias C, Robin S, Dray S (2019) Nine quick tips for analyzing network data. PLoS Comput Biol 15:e1007434.

Miller DR, Lebowitz JJ, Guenther DT, Refowich AJ, Hansen C, Maurer AP, Khoshbouei H (2019) Methamphetamine regulation of activity and topology of ventral midbrain networks. PLoS One 14:e0222957.

Morales M, Margolis EB (2017) Ventral tegmental area: cellular heterogeneity, connectivity and behaviour. Nat Rev Neurosci 18:73-85.

Mosharov EV, Larsen KE, Kanter E, Phillips KA, Wilson K, Schmitz Y, Krantz DE, Kobayashi K, Edwards RH, Sulzer D (2009) Interplay between cytosolic dopamine, calcium, and $\alpha$-synuclein causes selective death of substantia nigra neurons. Neuron 62:218-229.

Murakami M, Ishikura S, Kominami D, Shimokawa T, Murata M (2017) Robustness and efficiency in interconnected networks with changes in network assortativity. Appl Netw Sci 2:6.

Neuhoff H, Neu A, Liss B, Roeper J (2002) I(h) channels contribute to the different functional properties of identified dopaminergic subpopulations in the midbrain. J Neurosci 22:1290-1302.

Newman MEJ (2002) Assortative mixing in networks. Phys Rev Lett 89:208701.

Newman MEJ (2006) Finding community structure in networks using the eigenvectors of matrices. Phys Rev E Stat Nonlin Soft Matter Phys 74:036104.

Numan S, Seroogy KB (1999) Expression of trkB and trkC mRNAs by adult midbrain dopamine neurons: a double-label in situ hybridization study. J Comp Neurol 403:295-308.

O'Neill B, Patel JC, Rice ME (2017) Characterization of optically and electrically evoked dopamine release in striatal slices from digenic knock-in mice with DAT-driven expression of channelrhodopsin. ACS Chem Neurosci 8:310-319.

Onnela JP, Saramäki J, Kertész J, Kaski K (2005) Intensity and coherence of motifs in weighted complex networks. Phys Rev E Stat Nonlin Soft Matter Phys 71:065103.

Orlandi JG, Soriano J, Alvarez-Lacalle E, Teller S, Casademunt J (2013) Noise focusing and the emergence of coherent activity in neuronal cultures. Nature Phys 9:582-590.

Orsini CA, Colon-Perez LM, Heshmati SC, Setlow B, Febo M (2018) Functional connectivity of chronic cocaine use reveals progressive neuroadaptations in neocortical, striatal, and limbic networks. eNeuro 5: ENEURO.0081-18.2018.

Pachitariu M, Stringer C, Dipoppa M, Schröder S, Rossi LF, Dalgleish H, Carandini M, Harris KD (2017) Suite2p: beyond 10,000 neurons with 
standard two-photon microscopy. bioRxiv. Available at: https:// discovery.ucl.ac.uk/id/eprint/10049507/.

Palacios ER, Isomura T, Parr T, Friston K (2019) The emergence of synchrony in networks of mutually inferring neurons. Sci Rep 9:6412.

Paldino MJ, Zhang W, Chu ZD, Golriz F (2017) Metrics of brain network architecture capture the impact of disease in children with epilepsy. Neuroimage Clin 13:201-208.

Pandit R, Omrani A, Luijendijk MCM, de Vrind VAJ, Van Rozen AJ, Ophuis RJAO, Garner K, Kallo I, Ghanem A, Liposits Z, Conzelmann KK, Vanderschuren LJMJ, la Fleur SE, Adan RAH (2016) Melanocortin 3 receptor signaling in midbrain dopamine neurons increases the motivation for food reward. Neuropsychopharmacology 41:2241-2251.

Pernice V, Staude B, Cardanobile S, Rotter S (2011) How structure determines correlations in neuronal networks. PLoS Comput Biol 7:e1002059.

Pioli EY, Meissner W, Sohr R, Gross CE, Bezard E, Bioulac BH (2008) Differential behavioral effects of partial bilateral lesions of ventral tegmental area or substantia nigra pars compacta in rats. Neuroscience 153:1213-1224.

Pogarell O, Koch W, Karch S, Dehning S, Müller N, Tatsch K, Poepperl G, Möller H-J (2012) Dopaminergic neurotransmission in patients with schizophrenia in relation to positive and negative symptoms. Pharmacopsychiatry 45:S36-S41.

Poulin JF, Zou J, Drouin-Ouellet J, Kim KYA, Cicchetti F, Awatramani RB (2014) Defining midbrain dopaminergic neuron diversity by single-cell gene expression profiling. Cell Rep 9:930-943.

Poulin JF, Caronia G, Hofer C, Cui Q, Helm B, Ramakrishnan C, Chan CS, Dombeck DA, Deisseroth K, Awatramani R (2018) Mapping projections of molecularly defined dopamine neuron subtypes using intersectional genetic approaches. Nat Neurosci 21:1260-1271.

Prasad BM, Amara SG (2001) The dopamine transporter in mesencephalic cultures is refractory to physiological changes in membrane voltage. J Neurosci 21:7561-7567.

Pucak ML, Grace AA (1994) Evidence that systemically administered dopamine antagonists activate dopamine neuron firing primarily by blockade of somatodendritic autoreceptors. J Pharmacol Exp Ther 271:1181-1192.

Puopolo M, Raviola E, Bean BP (2007) Roles of subthreshold calcium current and sodium current in spontaneous firing of mouse midbrain dopamine neurons. J Neurosci 27:645-656.

Rayport S, Sulzer D, Shi WX, Sawasdikosol S, Monaco J, Batson D, Rajendran G (1992) Identified postnatal mesolimbic dopamine neurons in culture: morphology and electrophysiology. J Neurosci 12:4264-4280.

Reichardt J, Bornholdt S (2006) Statistical mechanics of community detection. Phys Rev E Stat Nonlin Soft Matter Phys 74:016110.

Rice ME (2000) Distinct regional differences in dopamine-mediated volume transmission. Prog Brain Res 125:277-290.

Rice ME, Patel JC (2015) Somatodendritic dopamine release: recent mechanistic insights. Philos Trans R Soc Lond B Biol Sci 370:20140185.

Rogers JH (1992) Immunohistochemical markers in rat brain: colocalization of calretinin and calbindin-D28k with tyrosine hydroxylase. Brain Res 587:203-210.

Rubinov M, Sporns O (2010) Complex network measures of brain connectivity: uses and interpretations. Neuroimage 52:1059-1069.

Saha K, Sambo D, Richardson BD, Lin LM, Butler B, Villarroel L, Khoshbouei H (2014) Intracellular methamphetamine prevents the dopamine-induced enhancement of neuronal firing. J Biol Chem 289:2224622257.

Sambo DO, Lin M, Owens A, Lebowitz JJ, Richardson B, Jagnarine DA, Shetty M, Rodriquez M, Alonge T, Ali M, Katz J, Yan L, Febo M, Henry LK, Bruijnzeel AW, Daws L, Khoshbouei H (2017) The sigma-1 receptor modulates methamphetamine dysregulation of dopamine neurotransmission. Nat Commun 8:2228.

Sandoval V, Hanson GR, Fleckenstein AE (2000) Methamphetamine decreases mouse striatal dopamine transporter activity: roles of hyperthermia and dopamine. Eur J Pharmacol 409:265-271.

Saunders BT, Richard JM, Margolis EB, Janak PH (2018) Dopamine neurons create Pavlovian conditioned stimuli with circuit-defined motivational properties. Nat Neurosci 21:1072-1083.
Schmitt KC, Reith MA (2010) Regulation of the dopamine transporter: aspects relevant to psychostimulant drugs of abuse. Ann NY Acad Sci 1187:316-340.

Schroeter MS, Charlesworth P, Kitzbichler MG, Paulsen O, Bullmore ET (2015) Emergence of rich-club topology and coordinated dynamics in development of hippocampal functional networks in vitro. J Neurosci 35:5459-5470.

Schultz W (1986) Responses of midbrain dopamine neurons to behavioral trigger stimuli in the monkey. J Neurophysiol 56:1439-1461.

Seroogy K, Ceccatelli S, Schalling M, Hökfelt T, Frey P, Walsh J, Dockray G, Brown J, Buchan A, Goldstein M (1988) A subpopulation of dopaminergic neurons in rat ventral mesencephalon contains both neurotensin and cholecystokinin. Brain Res 455:88-98.

Sporns O (2011) The non-random brain: efficiency, economy, and complex dynamics. Front Comput Neurosci 5:5.

Sporns O (2013) The structure and function of complex networks. Dialogues Clin Neurosci 15:247-262.

Sporns O, Kötter R (2004) Motifs in brain networks. PLoS Biol 2:e369.

Sporns O, Betzel RF (2016) Modular brain networks. Annu Rev Psychol 67:613-640.

Sporns O, Honey CJ, Kötter R (2007) Identification and classification of hubs in brain networks. PLoS One 2:e1049.

St Martin JL, Klucken J, Outeiro TF, Nguyen P, Keller-McGandy C, Cantuti-Castelvetri I, Grammatopoulos TN, Standaert DG, Hyman BT, McLean PJ (2007) Dopaminergic neuron loss and up-regulation of chaperone protein mRNA induced by targeted over-expression of alpha-synuclein in mouse substantia nigra. J Neurochem 100:1449-1457.

Starkweather CK, Uchida N (2020) Dopamine signals as temporal difference errors: recent advances. Curr Opin Neurobiol 67:95-105.

Stephans SE, Yamamoto BK (1995) Effect of repeated methamphetamine administrations on dopamine and glutamate efflux in rat prefrontal cortex. Brain Res 700:99-106.

Sulzer D (2007) Multiple hit hypotheses for dopamine neuron loss in Parkinson's disease. Trends Neurosci 30:244-250.

Sulzer D, Sonders MS, Poulsen NW, Galli A (2005) Mechanisms of neurotransmitter release by amphetamines: a review. Prog Neurobiol 75:406433.

Tarfa RA, Evans RC, Khaliq ZM (2017) Enhanced sensitivity to hyperpolarizing inhibition in mesoaccumbal relative to nigrostriatal dopamine neuron subpopulations. J Neurosci 37:3311-3330.

Telesford QK, Joyce KE, Hayasaka S, Burdette JH, Laurienti PJ (2011) The ubiquity of small-world networks. Brain Connect 1:367-375.

Teller S, Granell C, De Domenico M, Soriano J, Gómez S, Arenas A (2014) Emergence of assortative mixing between clusters of cultured neurons. PLoS Comput Biol 10:e1003796.

Thompson PM (2004) Structural abnormalities in the brains of human subjects who use methamphetamine. J Neurosci 24:6028-6036.

Tiklová K, Björklund ÅK, Lahti L, Fiorenzano A, Nolbrant S, Gillberg L, Volakakis N, Yokota C, Hilscher MM, Hauling T, Holmström F, Joodmardi E, Nilsson M, Parmar M, Perlmann T (2019) Single-cell RNA sequencing reveals midbrain dopamine neuron diversity emerging during mouse brain development. Nat Commun 10:581.

Tomasi D, Volkow ND, Wang R, Telang F, Wang G-J, Chang L, Ernst T, Fowler JS (2009) Dopamine transporters in striatum correlate with deactivation in the default mode network during visuospatial attention. PLoS One 4:e6102.

Vaillancourt DE, Spraker MB, Prodoehl J, Abraham I, Corcos DM, Zhou XJ, Comella CL, Little DM (2009) High-resolution diffusion tensor imaging in the substantia nigra of de novo Parkinson disease. Neurology 72:1378-1384.

van den Heuvel MP, de Lange SC, Zalesky A, Seguin C, Yeo BTT, Schmidt R (2017) Proportional thresholding in resting-state fMRI functional connectivity networks and consequences for patient-control connectome studies: issues and recommendations. Neuroimage 152:437-449.

van den Heuvel MP, Sporns O (2013) Network hubs in the human brain. Trends Cogn Sci 17:683-696.

van Wijk BCM, Stam CJ, Daffertshofer A (2010) Comparing brain networks of different size and connectivity density using graph theory. PLoS One 5: e13701.

Volkow ND, Morales M (2015) The brain on drugs: from reward to addiction. Cell 162:712-725. 
Volkow ND, Chang L, Wang GJ, Fowler JS, Franceschi D, Sedler M, Gatley SJ, Miller E, Hitzemann R, Ding YS, Logan J (2001a) Loss of dopamine transporters in methamphetamine abusers recovers with protracted abstinence. J Neurosci 21:9414-9418.

Volkow ND, Chang L, Wang GJ, Fowler JS, Leonido-Yee M, Franceschi D, Sedler MJ, Gatley SJ, Hitzemann R, Ding YS, Logan J, Wong C, Miller EN (2001b) Association of dopamine transporter reduction with psychomotor impairment in methamphetamine abusers. Am J Psychiatry 158:377-382.

Volkow ND, Wang GJ, Fowler JS, Thanos PPK, Logan J, Gatley SJ, Gifford A, Ding YS, Wong C, Pappas N, Thanos P (2002) Brain DA D2 receptors predict reinforcing effects of stimulants in humans: replication study. Synapse 46:79-82.

Wang GJ, Chang L, Volkow ND, Telang F, Logan J, Ernst T, Fowler JS (2004) Decreased brain dopaminergic transporters in HIV-associated dementia patients. Brain 127:2452-2458.
Wang HL, Morales M (2008) Corticotropin-releasing factor binding protein within the ventral tegmental area is expressed in a subset of dopaminergic neurons. J Comp Neurol 509:302-318.

Watts DJ, Strogatz SH (1998) Collective dynamics of "small-world" networks. Nature 393:440-442.

Yamada M, Bolden-Watson C, Watson MA, Cho T, Coleman NJ, Yamada M, Richelson E (1995) Regulation of neurotensin receptor mRNA expression by the receptor antagonist SR 48692 in the rat midbrain dopaminergic neurons. Brain Res Mol Brain Res 33:343-346.

Yu S, Huang D, Singer W, Nikolic D (2008) A small world of neuronal synchrony. Cereb Cortex 18:2891-2901.

Zalesky A, Fornito A, Cocchi L, Gollo LL, van den Heuvel MP, Breakspear M (2016) Connectome sensitivity or specificity: which is more important? Neuroimage 142:407-420. 